\title{
Coexistence of Multiple Points, Limit Cycles, and Strange Attractors in a Simple Autonomous Hyperjerk Circuit with Hyperbolic Sine Function
}

\author{
M. Fouodji Tsotsop $\mathbb{D}^{1,2}{ }^{1,2}$ J. Kengne, ${ }^{1}$ G. Kenne $\mathbb{D}^{\mathbb{D}},{ }^{1}$ and Z. Tabekoueng Njitacke $\mathbb{C}^{1,2}$ \\ ${ }^{1}$ Unité de Recherche d'Automatique et Informatique Appliquée (UR-AIA), Department of Electrical Engineering, \\ IUT-FV Bandjoun, University of Dschang (Cameroon), Dschang, Cameroon \\ ${ }^{2}$ Unité de Recherche de Matière Condensée, $d$ Electronique et de Traitement du Signal (UR-MACETS), Department of Physics, \\ University of Dschang, P.O. Box 67, Dschang, Cameroon
}

Correspondence should be addressed to M. Fouodji Tsotsop; tsotsopmerlinefouodji@yahoo.com

Received 18 December 2019; Revised 8 March 2020; Accepted 28 March 2020; Published 29 July 2020

Academic Editor: Oveis Abedinia

Copyright (c) 2020 M. Fouodji Tsotsop et al. This is an open access article distributed under the Creative Commons Attribution License, which permits unrestricted use, distribution, and reproduction in any medium, provided the original work is properly cited.

\begin{abstract}
In this contribution, a new elegant hyperjerk system with three equilibria and hyperbolic sine nonlinearity is investigated. In contrast to other models of hyperjerk systems where either hidden or self-excited attractors are obtained, the case reported in this work represents a unique one which displays the coexistence of self-excited chaotic attractors and stable fixed points. The dynamic properties of the new system are explored in terms of equilibrium point analyses, symmetry and dissipation, and existence of attractors as well. Common analysis tools (i.e., bifurcation diagram, Lyapunov exponents, and phase portraits) are used to highlight some important phenomena such as period-doubling bifurcation, chaos, periodic windows, and symmetric restoring crises. More interestingly, the system under consideration shows the coexistence of several types of stable states, including the coexistence of two, three, four, six, eight, and ten coexisting attractors. In addition, the system is shown to display antimonotonicity and offset boosting. Laboratory experimental measurements show a very good coherence with the theoretical predictions.
\end{abstract}

\section{Introduction}

Most authors have been interested in chaotic systems because of their sensitivity to the initial conditions and also to the variation of system parameters. Since the discovery of this phenomenon by Lorenz [1], many classical chaotic systems have emerged. We can mention the Rossler system [2], Chen system [3], Jafari system [4], Pham system [5], and Lü system just to name a few [6]. In the last few years, special attention has been given to "jerk systems" because of their simplicity and complex dynamics [7-12]. From a mathematical point of view, a generalization of the jerk dynamics is usually given in the following form:

$$
\frac{\mathrm{d}^{n} x}{\mathrm{~d} t^{n}}=f\left(\frac{\mathrm{d}^{n-1} x}{\mathrm{~d} t^{n-1}}, \ldots, \frac{\mathrm{d} x}{\mathrm{~d} t}, x\right)
$$

When $n=3$, we have $\left(\mathrm{d}^{3} x / \mathrm{d} t^{3}\right)=f\left(\left(\mathrm{~d}^{2} x / \mathrm{d} t^{2}\right)\right.$, $(\mathrm{d} x / \mathrm{d} t), x)$, which is called "jerk system" [13]. For $n \geq 4$, (1) turns to "hyperjerk system" or "snap system" [14]. In the literature, several authors have studied the latter. Generally, these systems exhibit multistability phenomenon which are the coexistence of multiple attractors solely depending on the initial conditions. These attractors are generally classified into two categories, namely, self-excited and hidden attractors [15-20]. Remember that self-excited attractors exist in systems with unstable equilibrium points [21-23]. In contrast, hidden attractors are characterized by the systems with no equilibrium [24-29], either by a line or a curve of 
equilibrium points, or system with stable equilibrium points $[24,25]$. In addition, hidden attractors have a basin of attraction which does not intersect with the neighborhoods of equilibria.

Interested by the self-excited attractors, many authors applied different techniques to hyperjerk systems. Some of these authors introduced different types of nonlinearities. For instance, in 2006, Cklouverakis and Sprott [22] presented a numerical study of a simple subclass of hyperjerk systems and showed that the $4^{\text {th }}$ and $5^{\text {th }}$ order hyperjerk systems developed some simple chaotic behaviors. In 2015, Sundiarapandian and coworkers [23] presented a new hyperchaotic 4-D hyperjerk system by adding a quadratic nonlinearity to the hyperjerk system of Chlouverakis-Sprott system. The authors present some qualitative and quantitative analyses of the new system. In 2017, Daltzis et al. [13] introduced a new hyperjerk system with two nonlinearities (absolute value and quintic term) and showed that the new system can develop hyperchaotic behaviors. Recently, Leutcho et al. [21] presented a new hyperjerk circuit with hyperbolic sine function and demonstrated that the novel proposed system is the unique one which is capable to exhibit the coexistence of nine periodic and chaotic attractors.

Motivated by the above mentioned results, we present a new hyperjerk system with nonlinear position feedback involving a hyperbolic sine function. Our circuit is derived from the hyperjerk system proposed by Dalkirian and Sprott [7] by replacing the exponential nonlinearity by the hyperbolic sine function. The striking aspect of the proposed system is its ability to develop the coexistence of up to ten disconnected attractors including periodic, chaotic, and point attractors. The objectives of this work are as follows: (a) to present an analytical study of the proposed hyperjerk system; (b) to highlight regions in which we observe the coexistence of multiple attractors; (c) to point out some striking features like antimonotonicity and offset boosting; and (d) to verify the feasibility of the proposed model through an experimental study.

This research is organized as follows. Section 2 deals with the modeling process. The electronic consuration of the hyperjerk circuit is presented and the suitable mathematical model is derived to describe the dynamics of the novel hyperjerk, wherein some basic properties of the model are equally presented. In Section 3, the bifurcation structures of the system are investigated numerically. Also, in this section, some tools are used to show multistability observed in the novel system. Section 4 contains experimental study, and at the end of this section, it appears that coherence is observed between the theoretical and experimental analysis. Finally, Section 5 presents conclusion.

\section{Description and Analysis of the Model}

2.1. Circuit Description. It is important to know that the new circuit proposed here derives from the hyperjerk system proposed by Dalkirian and Sprott [7]. It is obtained by substituting the exponential nonlinearity by the hyperbolic sine function. Figure 1 represents the schematic diagram of the novel hyperjerk circuit. The circuit consists of four successive integrators associated to several feedback loops. In addition, the nonlinear feedback loop linked with the pair of semiconductor diodes $\left(D_{1}, D_{2}\right)$ is applied to the first integrator. The symmetrical nature [30] of the system is due to the antiparallel configuration of the diodes. In such type of configuration, the voltage across each diode is equal to the voltage of the resulting two-terminal device, while the current is the addition of the currents flowing through each diode. The symmetrical property of the nonlinearity is necessary for the occurrence of symmetric attractors [30]. We would like to recall that the pair of semiconductor diodes is the only nonlinear element responsible for the chaotic behavior displayed by the whole electronic circuit.

2.2. State Equations. The following assumptions will be adopted throughout our analysis. Firstly, we considered that capacitors and operational amplifiers are ideal with the latter operating in linear domains. Secondly, the current-voltage characteristic (3) of the pair of semiconductor diodes $\left(D_{1}\right.$ and $\left.D_{2}\right)$ is obtained from the Shockley diode equation $[31,32]$ as follows:

$$
\begin{aligned}
I_{d}= & I_{D_{1}}-I_{D_{2}}=I_{S}\left[\exp \left(V_{d} / \eta V_{T}\right)-1\right] \\
& -I_{S}\left[\exp \left(-V_{d} / \eta V_{T}\right)-1\right]=2 I_{S} \sinh \left(V_{d} / \eta V_{T}\right),
\end{aligned}
$$

where $I_{S}, V_{T}=\left(k_{b} T / q\right), k_{b}, T, q$, and $\eta(1<\eta<2)$ are the intrinsic parameters of the diodes. By applying Kirchhoff's laws to Figure 1 and considering the above assumptions, it can be shown that the voltages $V_{1}, V_{2}, V_{3}$, and $V_{4}$ satisfy the following set of four coupled first-order nonlinear differential equations:

$$
\left\{\begin{array}{l}
C_{1} \frac{\mathrm{d} V_{1}}{\mathrm{~d} t}=\frac{V_{2}}{R} \\
C_{2} \frac{\mathrm{d} V_{2}}{\mathrm{~d} t}=\frac{V_{3}}{R_{m}} \\
C_{3} \frac{\mathrm{d} V_{3}}{\mathrm{~d} t}=\frac{V_{4}}{R_{d}}, \\
C_{4} \frac{\mathrm{d} V_{4}}{\mathrm{~d} t}=\frac{V_{1}}{R_{c}}-\frac{V_{2}}{R_{b}}-\frac{V_{3}}{R_{e}}-\frac{V_{4}}{R_{a}}-I_{d}
\end{array}\right.
$$

Applying the following change of variables:

$$
\begin{aligned}
t & =\tau R C, \\
V_{\text {ref }} & =10 \eta V_{T}, \\
x_{j} V_{\text {ref }} & =V_{j} \quad(j=1,2,3,4), \\
a & =R / R_{a}, \\
b & =R / R_{b}, \\
c & =R / R_{c} \\
d & =R / R_{d}, \\
m & =R / R_{m}, \\
e & =R / R_{e}, \\
\gamma & =2 R I_{S} / V_{\text {ref }},
\end{aligned}
$$




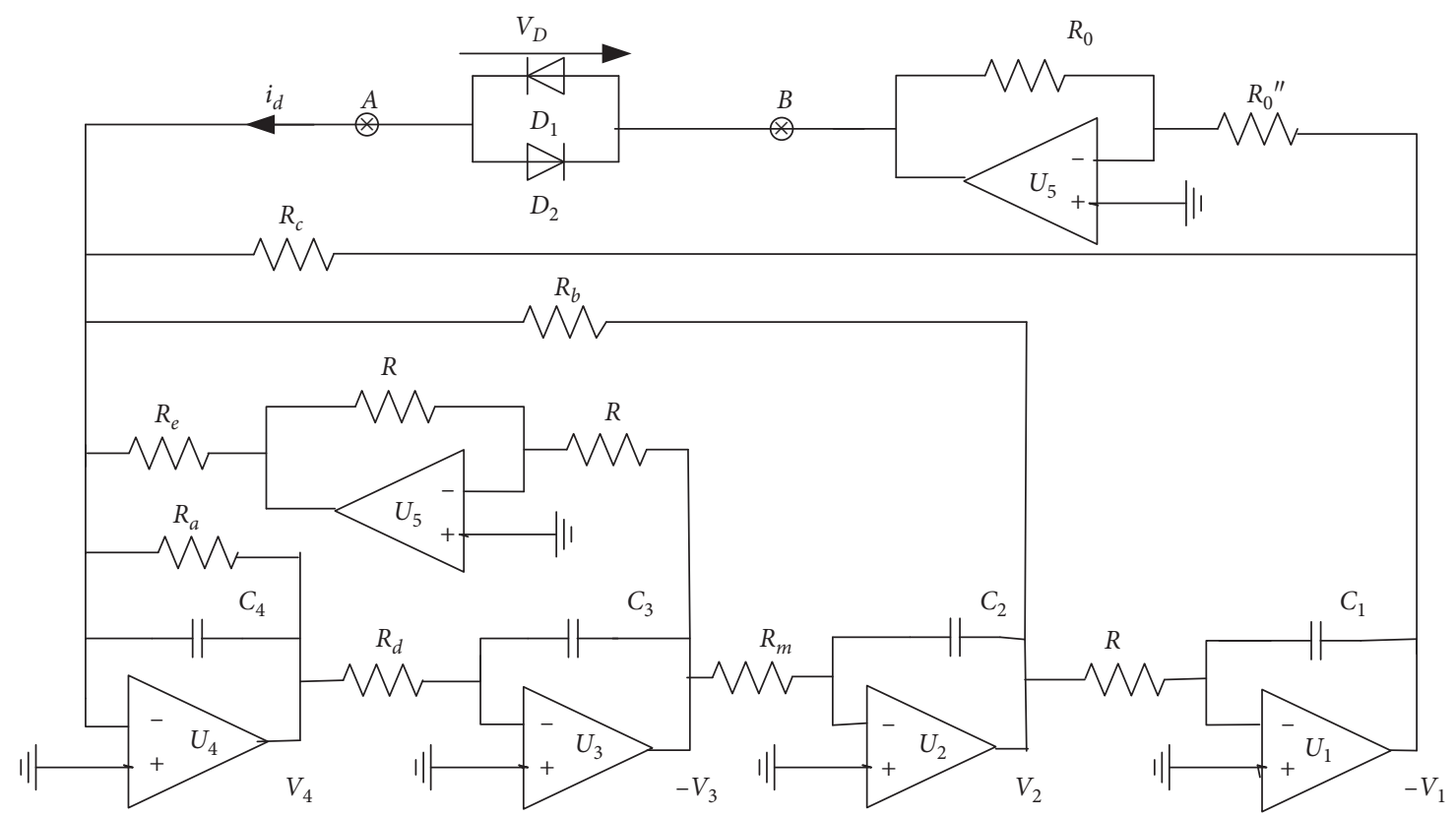

FIGURE 1: Electronic circuit realization of the novel 4-D hyperjerk system with hyperbolic sine nonlinearity. Its simplicity is remarkable. The pair of semiconductor diodes implements the hyperbolic nonlinearity of the model.

we get the normalized circuit equations which are expressed by the following smooth nonlinear fourth-order differential equations easy for numerical integration:

$$
\left\{\begin{array}{l}
\dot{x}_{1}=x_{2}, \\
\dot{x}_{2}=m x_{3}, \\
\dot{x}_{3}=d x_{4}, \\
\dot{x}_{4}=c x_{1}-b x_{2}-e x_{3}-a x_{4}-\gamma \sinh \left(x_{1}\right),
\end{array}\right.
$$

where the dot represents differentiation concerning the dimensionless time $\tau$. Note that the nonlinear function only depends on the state variable $x_{1}$ in system (5). $\gamma$ will be kept constant throughout the numerical analysis: $\gamma=0.0011$. Therefore, during the bifurcation analysis of the 4-D system, $c$ is considered like the control parameter (i.e., with respect to $R_{c}$ ). The values of electronic components used for both the numerical and experimental analyses are listed in Table 1. System (5) can be expressed equivalently in the general hyperjerk form as follows:

$$
\dddot{x}_{1}=m b c x_{1}-d \gamma \sinh \left(x_{1}\right)-m b \mathrm{~d} \dot{x}_{1}-d e \ddot{x}_{1}-a \dot{x}_{1} .
$$

By observing equation (6), it can be noticed that our model belongs to the wider class of "elegant" hyperjerk dynamical systems defined in [14]. More interestingly, our model (5) represents one of the simplest autonomous 4-D systems reported recently, displaying the coexistence of up to ten fixed points, periodic and chaotic attractors.

\subsection{Symmetry, Dissipation, and Existence of Attractors.} Equation (5) being invariant following the transformation $\left(x_{1}, x_{2}, x_{3}, x_{4}\right) \Longleftrightarrow\left(-x_{1},-x_{2},-x_{3},-x_{4}\right)$, we can conclude that we will have a couple of solutions for a given parameter
TABLE 1: List of electronic components used during the analyses.

\begin{tabular}{lcc}
\hline Components & \multicolumn{2}{c}{ Values } \\
\hline$R_{a}$ & $300 \Omega-1.7 k \Omega$ & $5.555 k \Omega$ \\
$R_{b}$ & $1.666 k \Omega$ & $2.631 k \Omega$ \\
$R_{c}$ & $10 k \Omega$ & $4 k \Omega-6 k \Omega$ \\
$R_{d}$ & $2 k \Omega$ & $7.407 k \Omega$ \\
$R_{e}$ & $5 k \Omega$ & $673.4 \Omega$ \\
$R_{m}$ & $10 k \Omega$ & $4.545 k \Omega$ \\
$R, R_{0}, R_{0}^{\prime \prime}$ (numerical study) & $10 k \Omega$ & $10 k \Omega$ \\
$R, R_{0}$ (experimental study) & $10 k \Omega$ & $10 k \Omega$ \\
$R_{0}^{\prime}($ experimental study) & $100 k \Omega$ & $100 k \Omega$ \\
$C_{1}, C_{2}, C_{3}, C_{4}$ & $10 n F$ & $10 n F$ \\
$D_{1}, D_{2}$ & $1 N 4148$ & $1 N 4148$ \\
\hline
\end{tabular}

range. So, if $\left(x_{1}, x_{2}, x_{3}, x_{4}\right)$ is a solution of our system, then its symmetry $\left(-x_{1},-x_{2},-x_{3},-x_{4}\right)$ will also be a solution. All these makes it possible to highlight the symmetrical nature of our system. In order to verify the dissipation property of our system, it is necessary to calculate the volume contraction rate $\left(\Lambda=\left(V^{-1} \mathrm{~d} V / \mathrm{d} t\right)\right)$. For every point of space $\left(x_{1}, x_{2}, x_{3}, x_{4}\right)^{T}$ $[33,34]$, it is given by the following expression:

$$
\Lambda=\frac{\partial \dot{x}_{1}}{\partial x_{1}}+\frac{\partial \dot{x}_{2}}{\partial x_{2}}+\frac{\partial \dot{x}_{3}}{\partial x_{3}}+\frac{\partial \dot{x}_{4}}{\partial x_{4}}=-a<0 .
$$

The above expression is negative and does not depend on the space coordinates of the system, and thus we can conclude that the introduced system is dissipative.

2.4. Fixed Point Analysis. By canceling the right side of equation (5), it is possible to determine the equilibrium 
points of the system which play a crucial role in the study of the system dynamics. The resolution of equation (8) permits to obtain different equilibrium points of the system.

$$
\left\{\begin{array}{l}
x_{2}=0 \\
x_{3}=0 \\
x_{4}=0 \\
c x_{1}-b x_{2}-e x_{3}-a x_{4}-\gamma \sinh \left(x_{1}\right)=0 .
\end{array}\right.
$$

Note that the point $E_{0}(0,0,0,0)$ is a trivial equilibrium point, while $E_{1}$ and $E_{2}$ are the solutions of the transcendental equation:

$$
c x_{1}-\gamma \sinh \left(x_{1}\right)=0 \text {. }
$$

By fixing $c=2.442$ and maintaining $\gamma$ at the same previous value, we obtain the other nontrivial equilibrium points $\left(E_{1}, E_{2}\right)=( \pm 10.79,0,0,0)$. The stability of the system in the neighborhood of those equilibrium points is studied by resolving the characteristic equation (12), resulting from the below equation:

$$
\operatorname{det}\left(M_{J}-\lambda I_{d}\right)=0
$$

where $I_{d}$ represents the $4 \times 4$ identity matrix and $M_{J}$ the Jacobian matrix defined as follows:

$$
M_{J}=\left[\begin{array}{cccc}
0 & 1 & 0 & 0 \\
0 & 0 & m & 0 \\
0 & 0 & 0 & d \\
c-\gamma \cosh \left(x_{1}^{0}\right) & -b & -e & -a
\end{array}\right] .
$$

The characteristic equation obtained is $\lambda^{4}+c_{3} \lambda^{3}+c_{2} \lambda^{2}+$ $c_{1} \lambda+c_{0}=0$, with

$$
\begin{aligned}
& c_{0}=-\operatorname{md}\left(c-\gamma \cosh \left(x_{1}^{0}\right)\right), \\
& c_{1}=\operatorname{mdb}, \\
& c_{2}=\mathrm{ed}, \\
& c_{3}=a .
\end{aligned}
$$

By applying the Lyapunov stability theory, it is shown that at the equilibrium point $E_{0}(0,0,0,0)$, $c-\gamma>0 \Longrightarrow c_{0}<0$, and thus the equilibrium $E_{0}$ is unstable since the characteristic equation has coefficients with different signs. In contrast, the stability of the nonzero equilibrium points $\left(E_{1,2}( \pm 10.79,0,0,0)\right)$ depends on the control parameters $m$ and $b$. By applying the Routh-Hurwitz criterion $[32,33]$, we have shown that for $b<b_{c 1}=3.4397$ and $b>b_{c 2}=5.56$, the nontrivial equilibrium points are unstable, but for $b_{c 1}<b<b_{c 2}$, they are stable. These critical values are obtained from the calculation of the Hopf bifurcation conditions:

$$
\begin{aligned}
\omega_{\mathrm{Hopf}} & =\sqrt{(\mathrm{mdb} / a)}, \\
b_{c 1, c 2} & =\frac{1}{2}\left(\frac{e a}{m} \pm \sqrt{\Delta}\right),
\end{aligned}
$$

with $\Delta=(\mathrm{ea} / m)^{2}+\left(4 a^{2} / \mathrm{md}\right)(c-\cosh (10.79))$.
Equation (13) provides the frequency of stable oscillations as well as the critical values of $b_{c 1, c 2}$ corresponding to the Hopf bifurcation of the system. From Table 2, it follows that in the regime of (periodic or chaotic) oscillations, the three equilibria are unstable, and thus the system generates self-excited oscillations. For the following parameters $a=1.8, d=1.35, e=15, m=3$, and $c=2.442$, the equilibrium point $E_{0}(0,0,0,0)$ remains unstable for all values of control parameter $b$. Moreover, for some values of bifurcation parameter $b$, the nontrivial equilibria have pure imaginary roots, and thus the system presents the Hopf bifurcation. In order to verify the existence of the Hopf bifurcation in the system, eigenvalue locus is plotted. It shows the existence of Hopf bifurcation in the system which is characterized by the intersection of the eigenvalue locus with the imaginary axis. By observing Figures 2(a) and 2(b), we can certify that the new hyperjerk system presents Hopf bifurcation.

\section{Numerical Computation}

3.1. Numerical Techniques. System (5) is resolved numerically in order to highlight the rich variety of bifurcation that can be observed in a new hyperjerk system. The dynamic properties of the model were numerically simulated in Turbo Pascal using the fourth-order Runge-Kutta method with a constant time step size of $2 \times 10^{-3}$, and parameters are taken in extended precision mode. The transient phase is canceled by integrating the system for a long time. The bifurcation diagram and the Lyapunov exponent are the traditional tools that measure the dependence of the system on the initial conditions as well as the sequence that leads to chaos in the system. The algorithms of Wolf and his collaborators [35] are used for calculating the Lyapunov exponents.

3.2. Bifurcation, Chaos in a Novel Hyperjerk Circuit. Different scenarios exhibited by the proposed hyperjerk system are obtained by plotting the bifurcation diagrams. The bifurcation diagram of Figure 3(a) is obtained by plotting the local maxima of the variable $x_{1}$ according to the bifurcation parameter $a$, the other parameters being fixed at $c=1, b=6, d=5, e=2$, and $m=1$. It can be noted that it is a period-doubling route to chaos because the transition from period-1 attractor to double-band chaos is as follows: period-1 $\longrightarrow$ period-2 $\longrightarrow$ period- $\longrightarrow$ single-band chaos $\longrightarrow$ period-5 $\longrightarrow$ single-band chaos $\longrightarrow$ doubleband chaos. Figure 4 clearly shows the above transition. It is obtained by progressively varying the control parameter. The exact nature of the attractors mentioned above is defined by the graphs of the four largest Lyapunov exponents shown in Figure 3(b). We can observe in Figure 3(b) that periodic attractors are characterized by $\lambda_{1}=0, \lambda_{2}, \lambda_{3}$, and $\lambda_{4}<0$, while chaotic attractors have the following characteristics: $\lambda_{1}>0$, $\lambda_{2}=0, \lambda_{3}$, and $\lambda_{4}<0$. A perfect coherence is observed between the bifurcation diagram and the corresponding graphs of the four largest Lyapunov exponents. In order to show the complexity of the new hyperjerk circuit, the chaotic attractor has been projected on several planes (Figures 5(a)-5(f)), as well 
TABLE 2: Corresponding eigenvalues of each equilibrium point according to the bifurcation parameter $b$.

\begin{tabular}{lcc}
\hline $\begin{array}{l}\text { Values of bifurcation parameter } \\
b\end{array}$ & $\begin{array}{c}\text { Eigenvalues at nontrivial fixed }\left(E_{1}, E_{2}\right), \lambda_{1}, \lambda_{2}, \lambda_{3}, \lambda_{4} \\
\text { Eigenvalues at the origin } E_{0}(0,0,0,0) \\
\lambda_{1}, \lambda_{2}, \lambda_{3}, \lambda_{4}\end{array}$ \\
\hline 3.1 & $-0.9641 \pm 3.4906 i 0.0641 \pm 2.7165 i$ (unstable) & $-0.5719 \pm 4.4347 i-1.10410 .4478$ (unstable) \\
3.2 & $-0.9461 \pm 3.4712 i 0.0461 \pm 2.7346 i$ (unstable) & $-0.5618 \pm 4.4350 i-1.11850 .4422$ (unstable) \\
3.4397 & $-0.9000 \pm 3.4206 i \pm 2.7820 i$ (neutral, & $-0.5378 \pm 4.4358 i-1.15360 .4292$ (unstable) \\
3.5 & Hopf bifurcation) & $-0.5318 \pm 4.4361 i-1.16250 .4260$ (unstable) \\
5.55 & $-0.0023 \pm 3.5339 i-0.8977 \pm 2.6358 i$ (stable) & $-0.3297 \pm 4.4591 i-1.47570 .3351$ (unstable) \\
5.56 & $-0.0093 \pm 3.5246 i-0.8907 \pm 2.6459 i$ (stable) & $-0.3287 \pm 4.4593 i-1.47720 .3347$ (unstable) \\
5.59 & Hopf bifurcation) & $-0.3258 \pm 4.4598 i-1.48190 .3336$ (unstable) \\
9.12 & $-0.9476 \pm 3.2070 i 0.0476 \pm 3.0071 i$ (unstable) & $-0.0077 \pm 4.5497 i-2.02100 .2363$ (unstable) \\
\hline
\end{tabular}

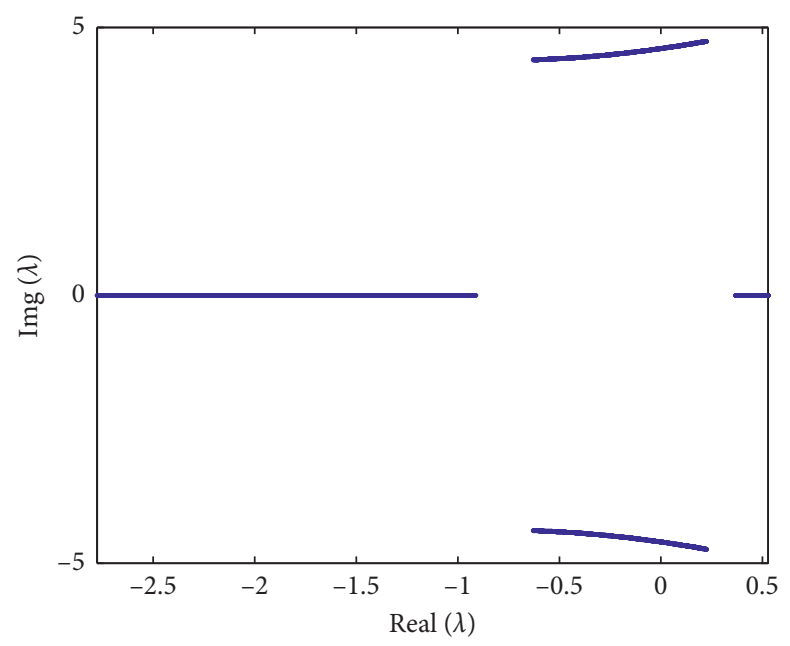

(a)

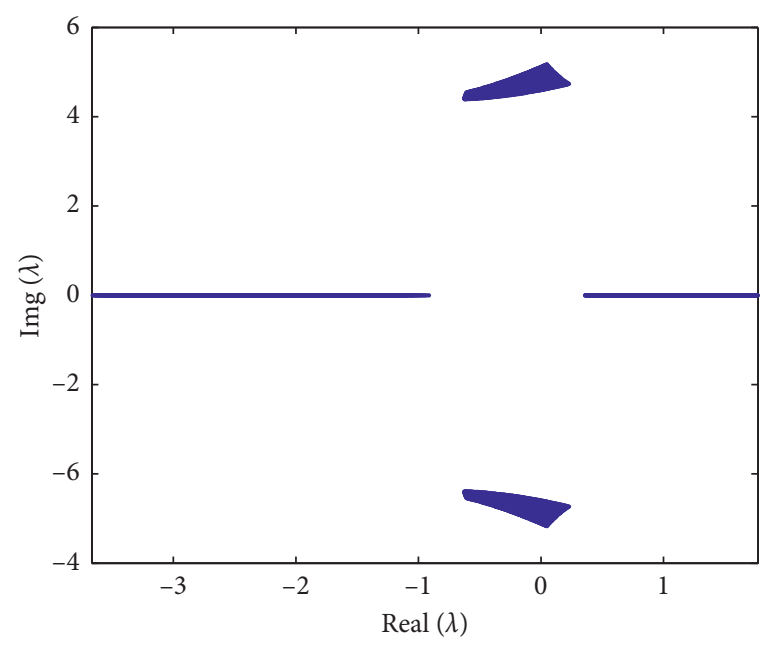

(b)

FIGURE 2: Representation of eigenvalue locus in the complex plan $(\operatorname{Re}(\lambda), \operatorname{Im}(\lambda))$ with the following parameter values: (a) $a=1.8, b=3.8$, $c=2.442, d=1.35, e=14.85$, and $2 \leq m \leq 10$; (b) $a=1.8, b=3.8, d=1.35, e=14.85,2 \leq m \leq 10$, and $2.34 \leq c \leq 12.985$. The appearance of eigenvalues in complex conjugate pairs justifies the symmetry observed in the system and intersection of the curve with imaginary axis shows the presence of the Hopf bifurcation in the system.

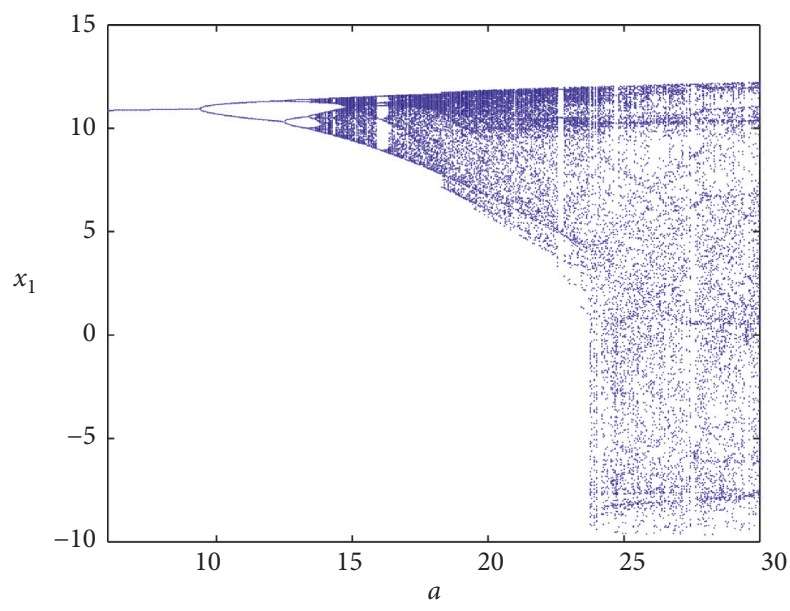

(a)

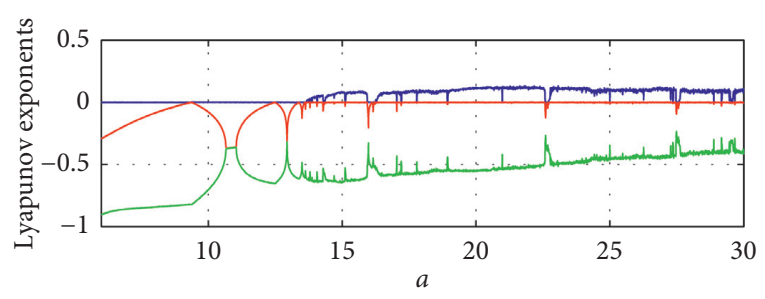

(b)

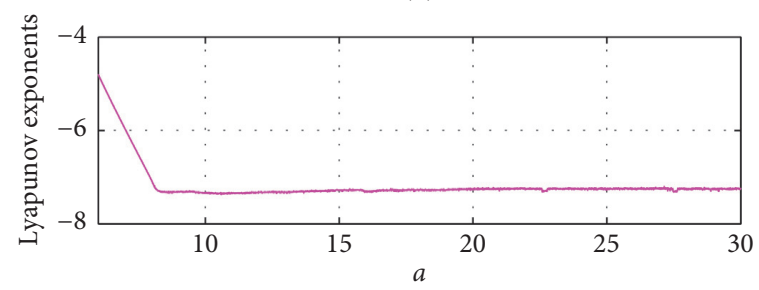

(c)

FIGURE 3: (a) Bifurcation diagram showing local maxima of the coordinate $x_{1}$ versus $c$ and $(\mathrm{b}, \mathrm{c})$ the corresponding graphs of four largest Lyapunov exponents plotted in the range $6 \leq a \leq 30$, with $c=1, b=6, d=5, e=2$, and $m=1$ and initial conditions $\left(x_{1}(0), x_{2}(0), x_{3}(0), x_{4}(0)\right)=(0,0,2.4,0)$. 


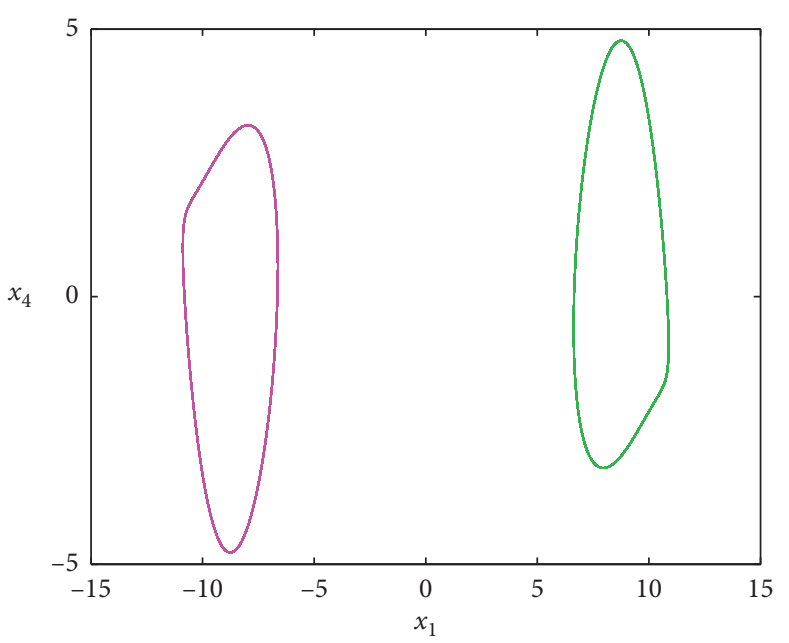

(a)

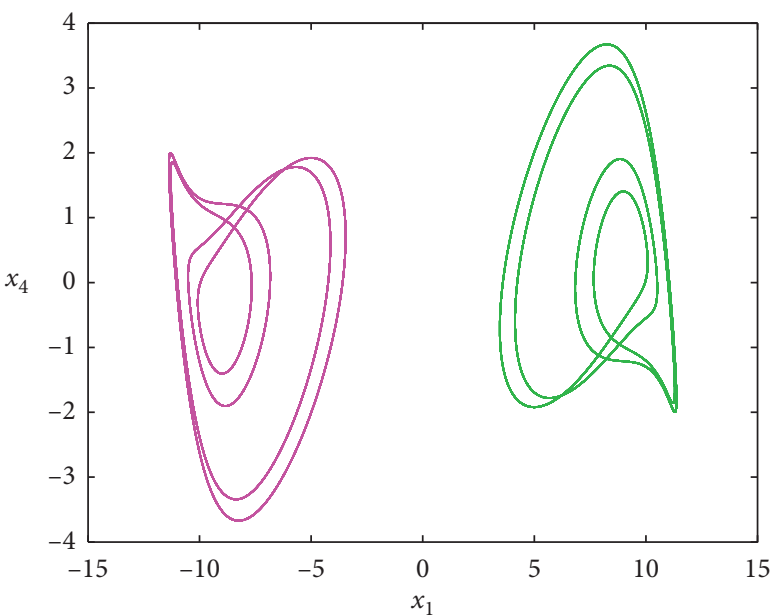

(c)

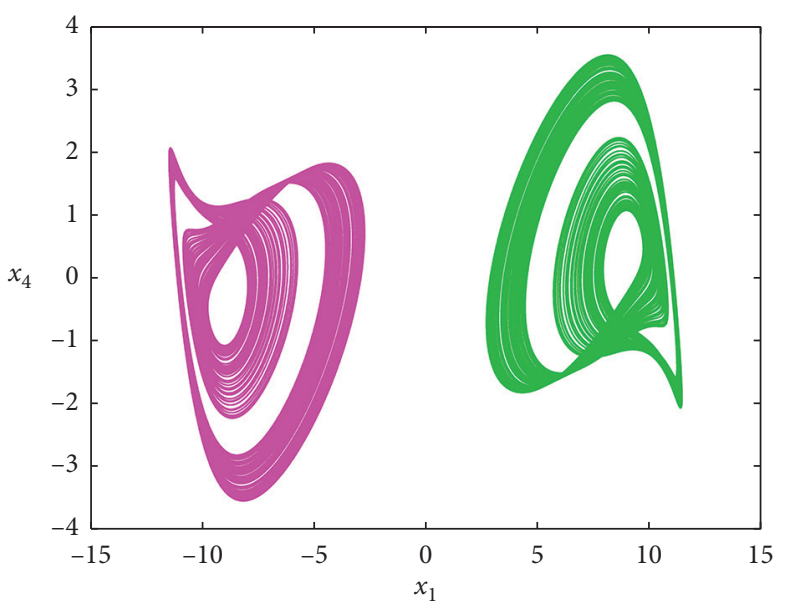

(e)

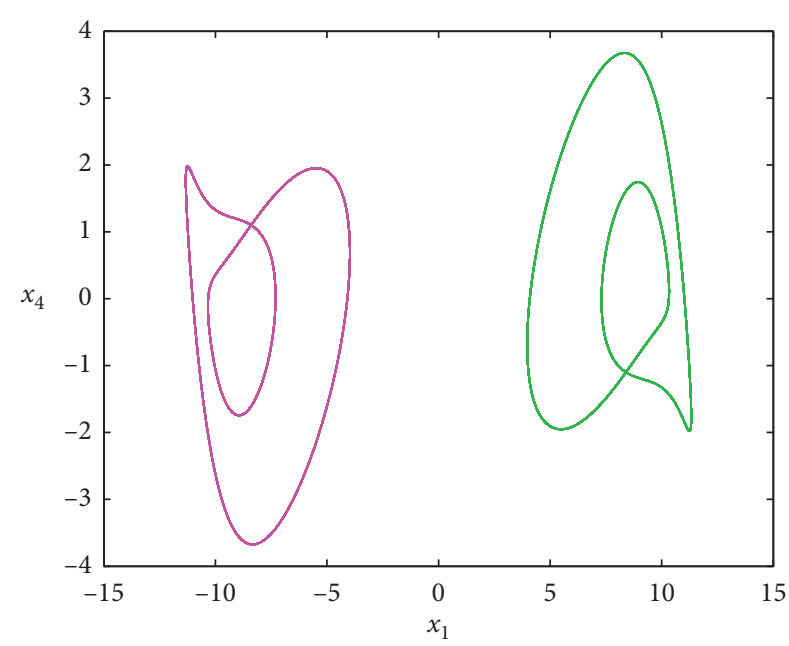

(b)

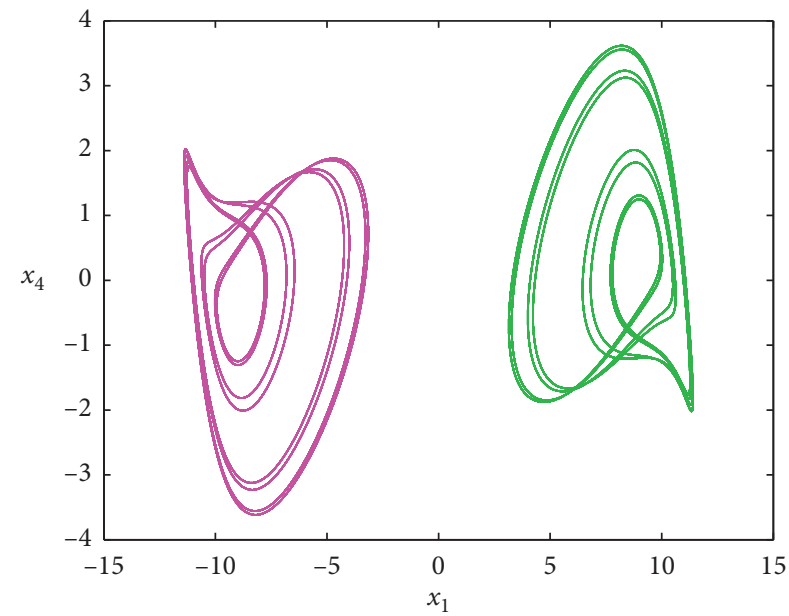

(d)

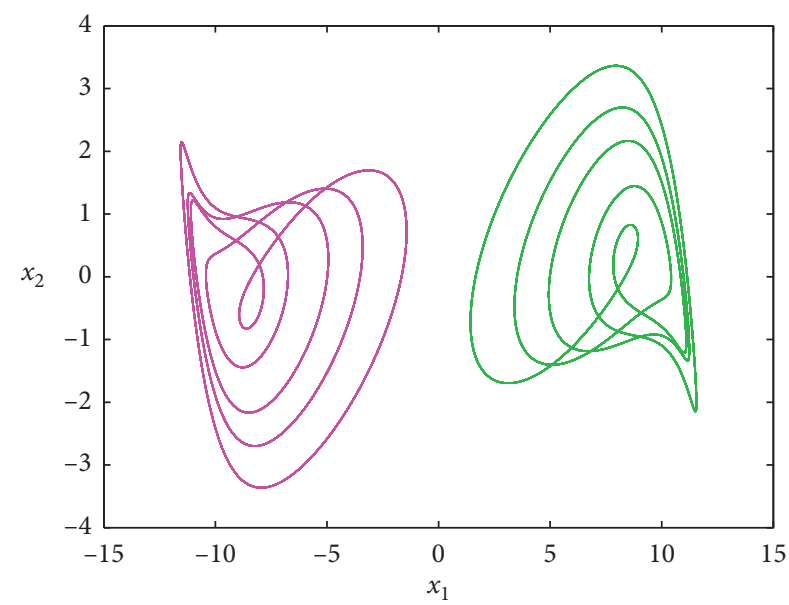

(f)

Figure 4: Continued. 


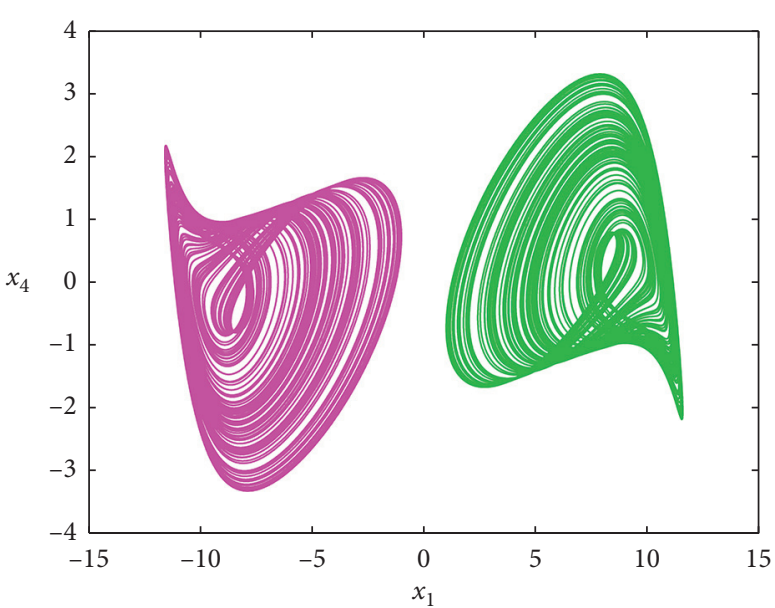

(g)

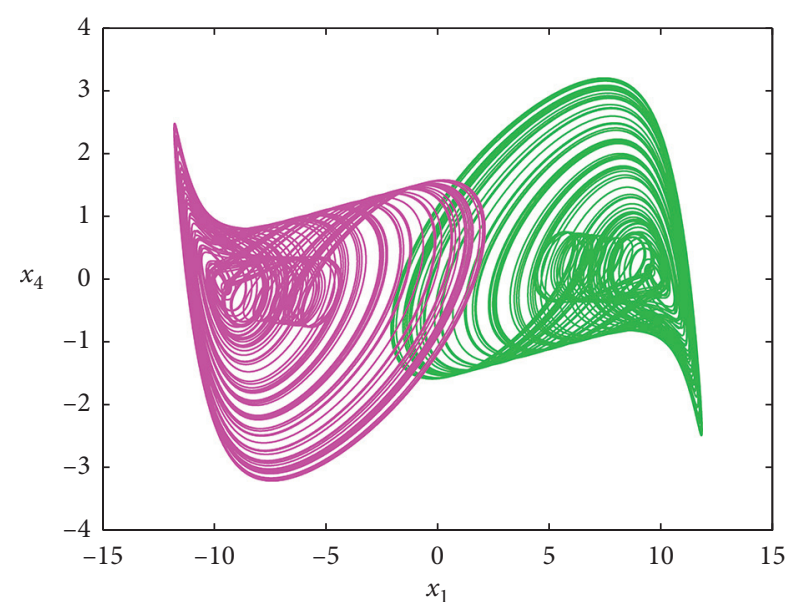

(h)

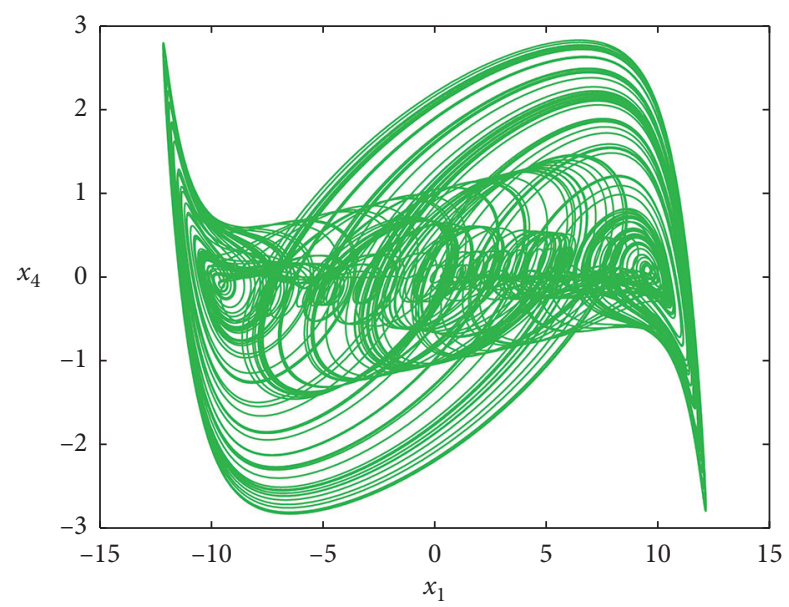

(i)

FIGURE 4: Numerical phase space trajectories showing routes to chaos in the system when varying the control parameter $c$ : (a) period-1 for $a=7$, (b) period-2 for $a=12.38$, (c) period-4 for $a=13$, (d) period-8 for $a=13.49$, (e) single-band chaos for $a=14.6$, (f) period-5 for $a=16.08$, (g) single -band chaos for $a=16.7$, (h) single-band chaos for $a=20$, and (h) double-band chaos for $a=30$. Initial conditions $\left(x_{1}(0), x_{2}(0), x_{3}(0), x_{4}(0)\right)$ are $(0,0,2.4,0)$. The others parameters are fixed as follows: $c=1, b=6, d=5, e=2$, and $m=1$.

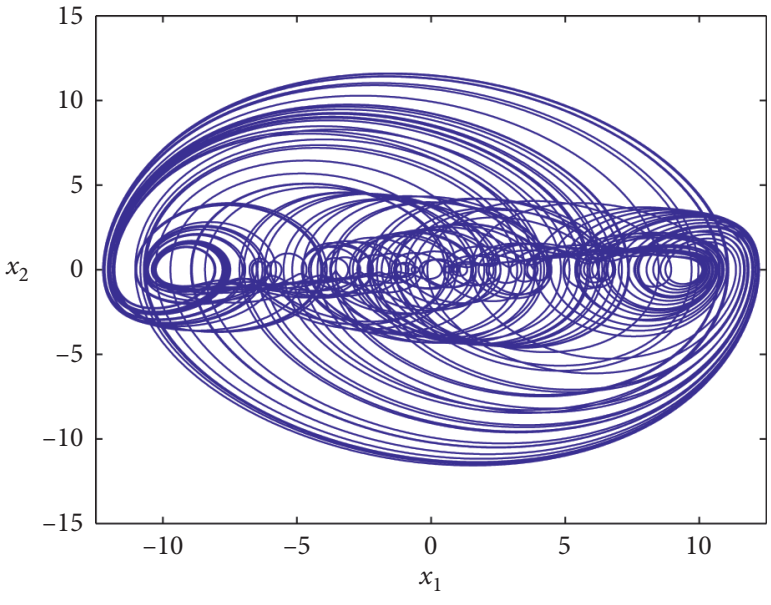

(a)

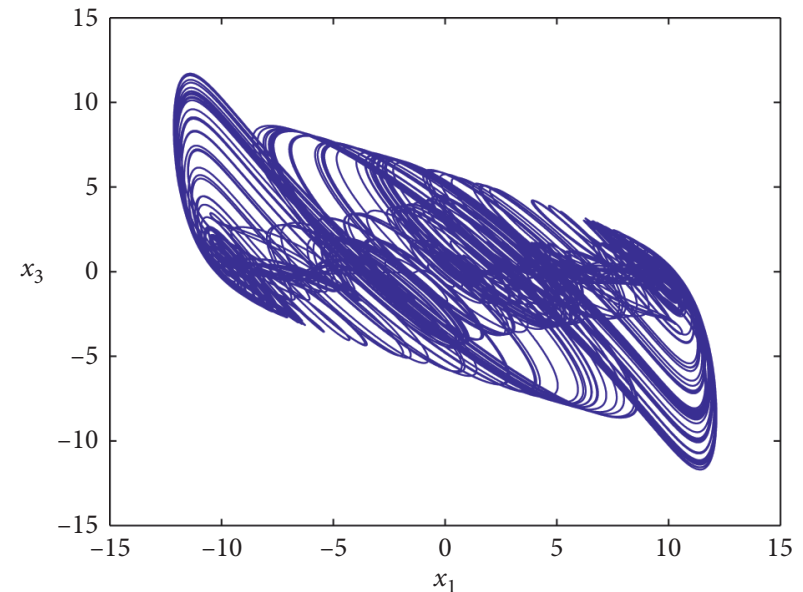

(b)

Figure 5: Continued. 


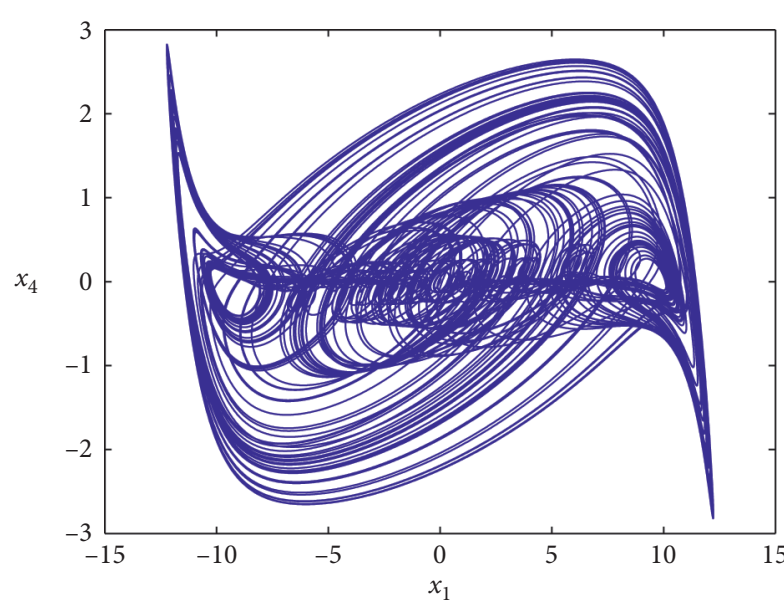

(c)

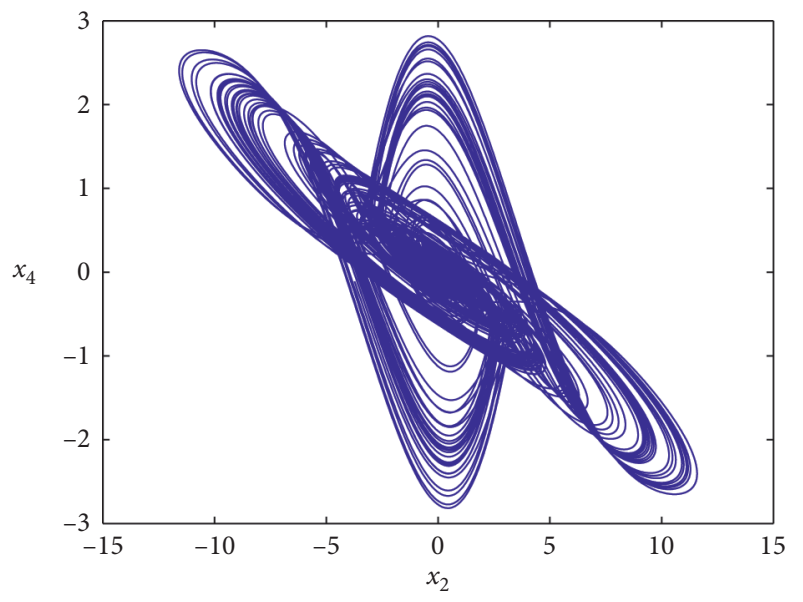

(e)

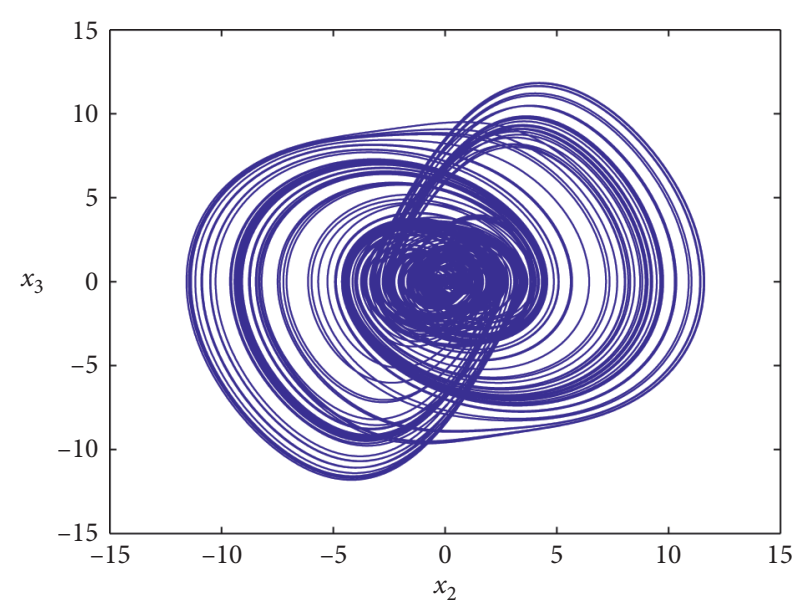

(d)

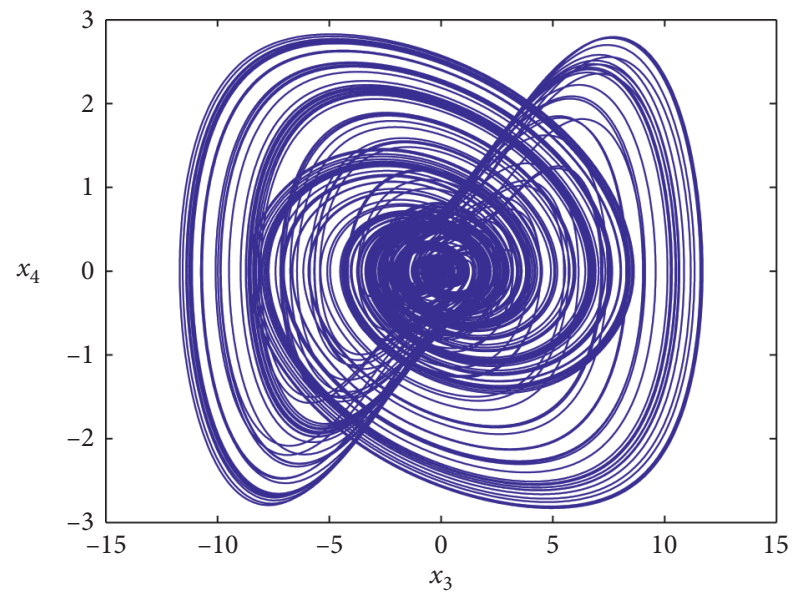

(f)

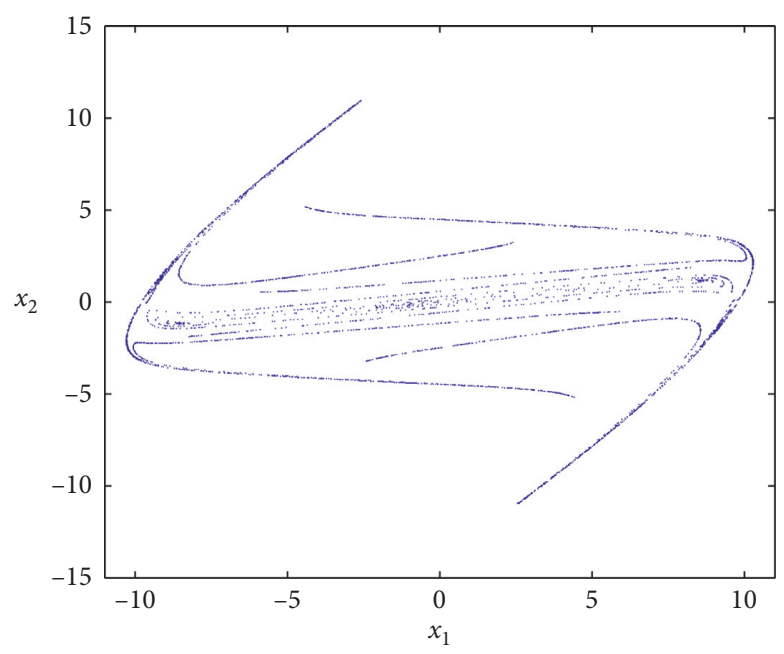

(g)

Figure 5: 2-D projection of the phase portraits of symmetric double-band chaotic attractors (a-f) of system (3) plotted into planes $\left(x_{1}-x_{4}\right)$, $\left(x_{2}-x_{3}\right),\left(x_{2}-x_{4}\right),\left(x_{3}-x_{4}\right)$ and corresponding double-sided Poincaré section $(e)$ in the plane $x_{1}=0$. Parameters are the same as those in Figure 4 .

as the Poincaré section (Figure 5(g)). We can observe that the double-band chaos completely changes when moving from one plane to another. For the value of the bifurcation parameter $a=17.04$, the coexistence of four periodic and chaotic attractors is observed in the novel proposed system (see Figure 6). In order to illustrate the Hopf bifurcation previously 


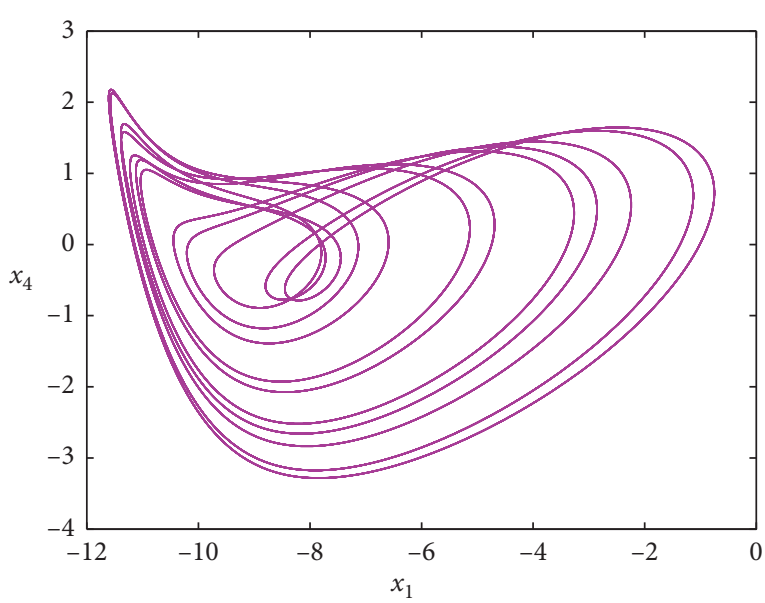

(a)

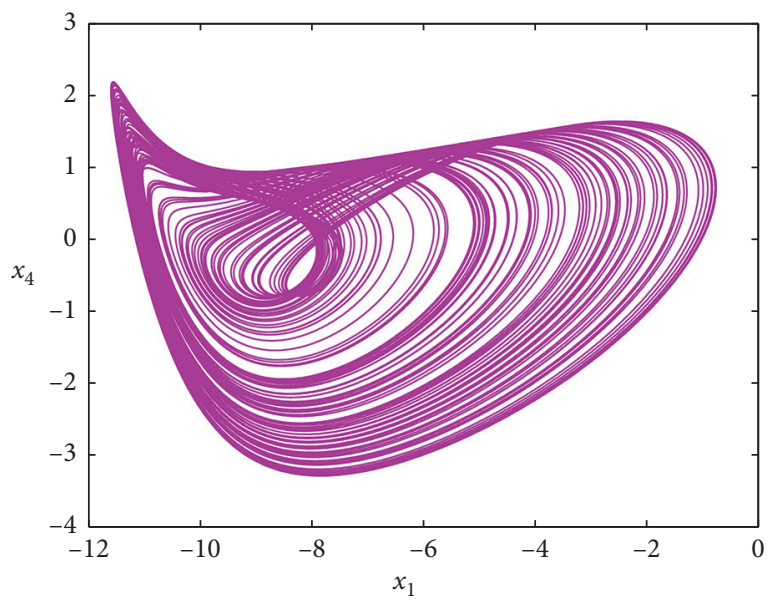

(c)

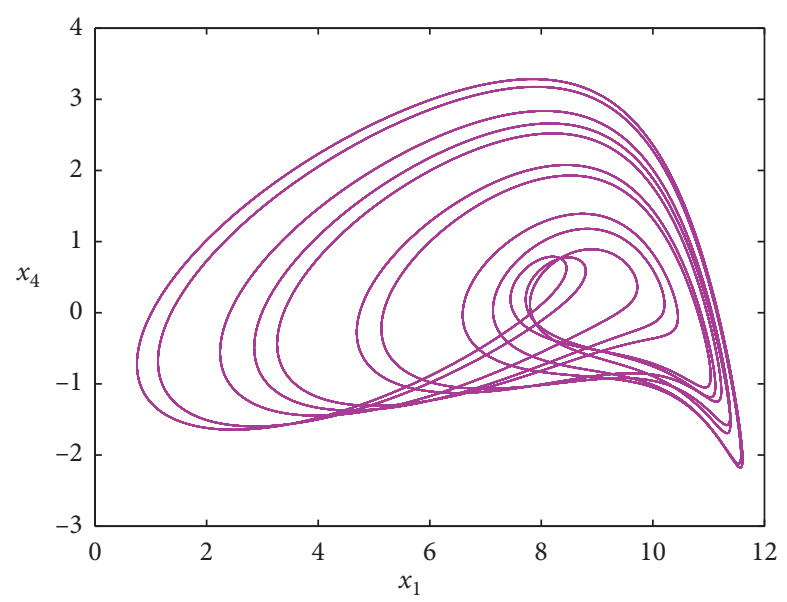

(b)

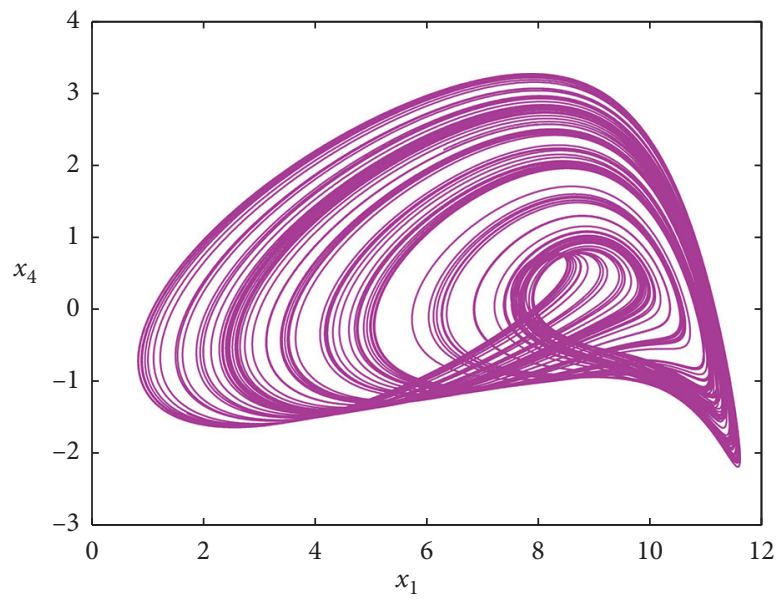

(d)

FIgURE 6: Coexistence of four attractors (a pair of period-11 limit cycle and a pair of chaotic attractors) with $a=17.04$, and their corresponding initial conditions are $( \pm 4.8,0,0,0)$ and $( \pm 1.2,0,0,0)$.

proved by theoretical calculations, the bifurcation diagram of Figure 7 has been represented. Stable state is characterized by a fixed point with $\lambda_{\max }<0$, while oscillatory state is characterized by $\lambda_{\max } \geq 0$.

3.3. Multistability. In this section, we demonstrate the variety of dynamical regimes in the new 4-D system. We show that depending on the values of the system parameters, the system exhibits very rich dynamics and bifurcation scenarios. A multistable system is a system with various coexisting stable states (chaotic, point, and periodic state) under the same system parameters, with different initial conditions. In recent years, the phenomenon of multistability phenomenon has been reported in many nonlinear dynamic systems [13, 36-46].

3.3.1. Coexistence of Attractors with respect to Bifurcation Parameter $c$. By changing the system parameters and considering $c$ as bifurcation parameter, we observe a completely different behavior. In addition, a very interesting phenomenon which is the coexistence of multiple attractors appears in the new 4-D hyperjerk. For this phenomenon to be illustrated, the bifurcation diagrams of Figure 8 are plotted using the following method:

(i) The blue diagram is obtained by simultaneously increasing the value of the control parameter $c$ as well as the initial condition $x(0)$. At each iteration, we assign to $x(0)$ the new value of the control parameter $c$.

(ii) The red diagram is obtained by incrementing $c$ from its minimum value 2.34 to its maximum value 2.985 , with a carefully chosen step. Note that the solutions of the system at each iteration are considered as the initial condition of the next iteration.

(iii) The cyan diagram respects the previous procedure, with the initial condition $(-10.67,0,0,0)$, whereas the black diagram follows the same procedure as previously described but the only difference is the decrease of the control parameter $c$. The initial condition is $(10,0,0,0)$. 


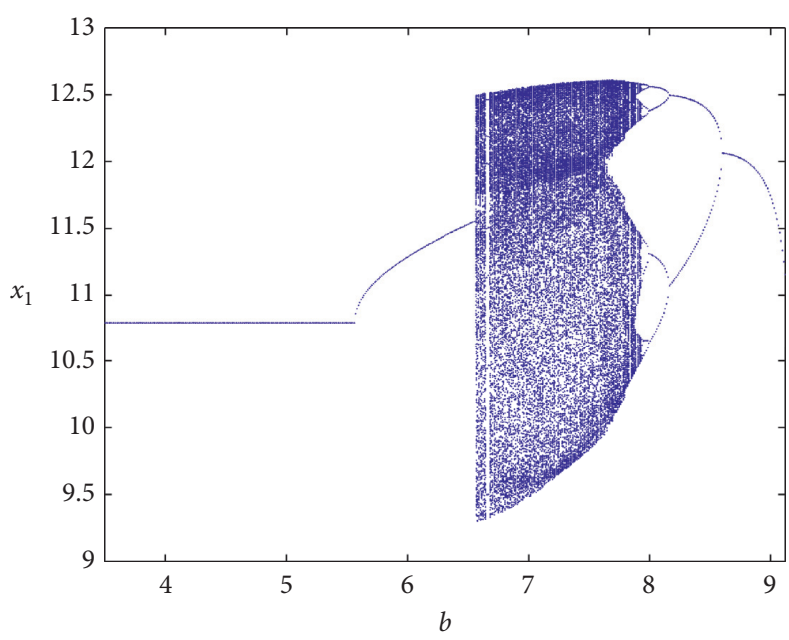

(a)

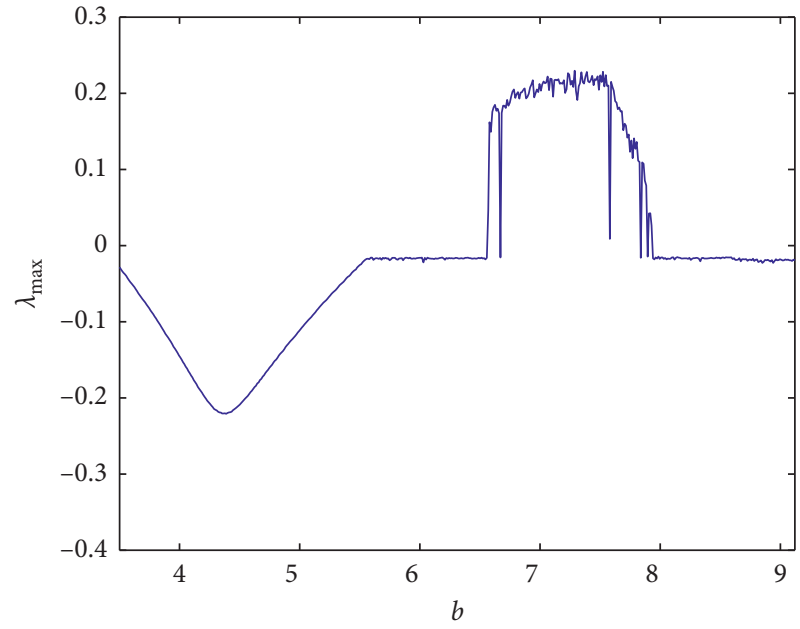

(b)

Figure 7: (a) Bifurcation diagram showing local maxima of the coordinate $x_{1}$ versus $b$ and (b) the corresponding graph of largest Lyapunov exponent $\left(\lambda_{\max }\right)$ plotted by decreasing $b$ in the range $3.5 \leq b \leq 9.12$, with $a=1.8, m=3, c=2.442, d=1.35$, and $e=15$ and initial conditions $\left(x_{1}(0), x_{2}(0), x_{3}(0), x_{4}(0)\right)=(0,0,9.333,0)$.

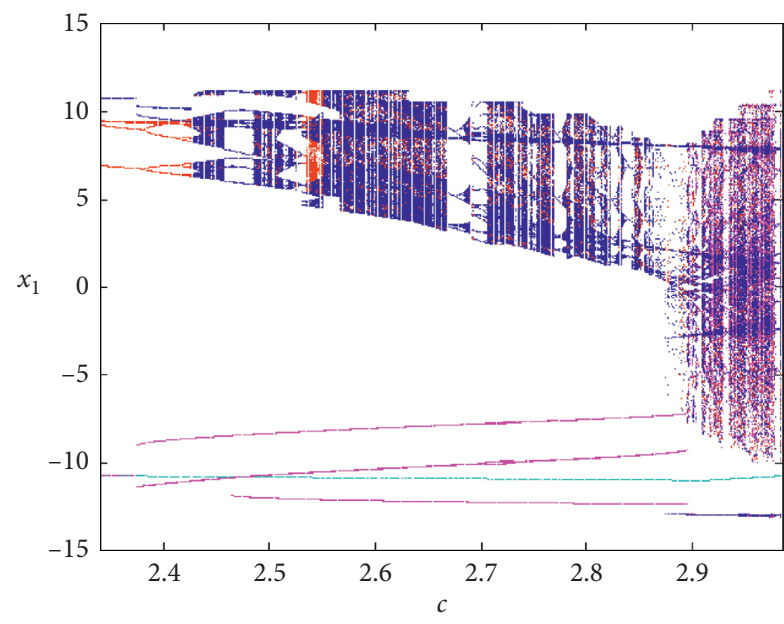

(a)

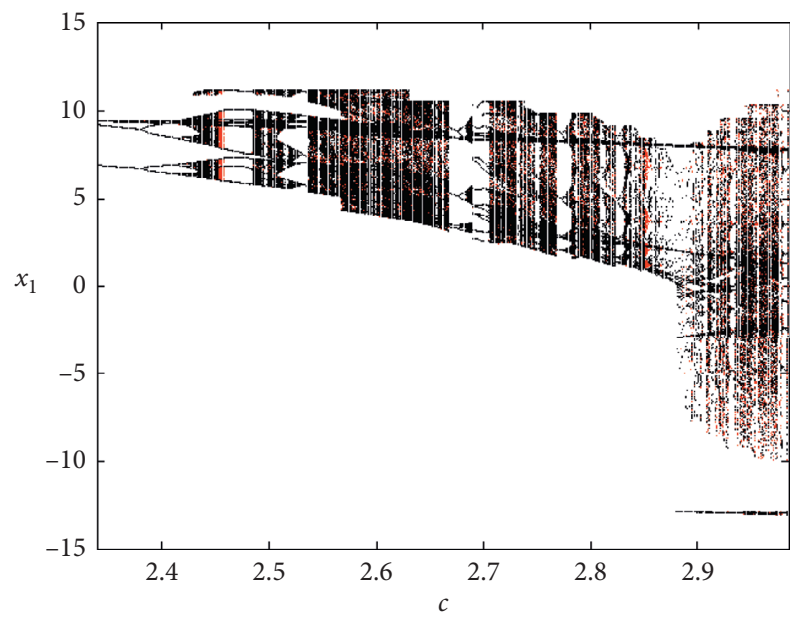

(b)

FIGURE 8: symmetric coexisting bifurcation diagrams showing complex structure of the new hyperjerk for $a=1.8, b=3.8, d=1.35, e=14.85$, and $m=2$.2. Initial conditions $y(0)=z(0)=w(0)=0$ and $x(0)$ are fixed as follows for obtaining these diagrams: red diagrams for increasing $c$ with $x(0)=5.25$, black diagram for decreasing $c$ with $x(0)=-10$, cyan diagrams for increasing $c$ with $x(0)=-10.67$, magenta diagram for increasing and decreasing, started at $c=2.5442$ with $x(0)=-4$, and blue diagram for starting $c$ with same initial condition $x(0)=c$. For more information, see Table 3.

(iv) The magenta diagram is obtained by increasing the control parameter $c$ from 2.398 to 2.985 , followed by decreasing the bifurcation parameter $c$ from 2.398 to 2.34 .

We can observe in Figure 8 several windows of coexisting attractors. For more details about the methods used to plot the bifurcation diagrams of Figure 8, see Table 3. The enlarged bifurcation diagram of Figure 9 shows the hysteretic domain, plotted in the range $2.442 \leq c \leq 2.488$, and the techniques used to plot the diagrams are also presented in
Table 3. Figure 9 shows the coexistence of six and eight different limit cycles, chaotic and point attractors. Some sample phase portraits showing the coexistence of six and eight attractors are presented in Figures 10 and 11, respectively. Some basins of attractions showing the initial conditions domains of the coexisting attractors are presented in Figure 12. The coexistence of four attractors is clearly denoted (a pair of periodic attractors (black and yellow) and a pair of chaotic attractors (blue and green)). Note that there is a perfect symmetry between the different cross sections of the competing attractors. 
TABle 3: Techniques used to obtain coexisting bifurcation diagrams and corresponding initial conditions.

\begin{tabular}{lcccc}
\hline Fig. $n^{0}$ & Color graph & Parameter range & Sweeping direction & Initial condition $\left(x_{1}(0), x_{2}(0), x_{3}(0), x_{4}(0)\right)$ \\
\hline & Blue & $2.34 \leq c \leq 2.985$ & Upward & $(c, 0,0,0)$ \\
Red & $2.34 \leq c \leq 2.985$ & Upward & $(5.25,0,0,0)$ \\
Figure 8 & Cyan & $2.34 \leq c \leq 2.985$ & Upward & $(-10.67,0,0,0)$ \\
& Black & $2.985 \leq c \leq 2.34$ & Downward & $(-10,0,0,0)$ \\
& Magenta & $2.398 \leq c \leq 2.985$ & Upward & $(-4,0,0,0)$ \\
\hline & $2.398 \leq c \leq 2.34$ & Downward & $(5,0,0,0)$ \\
Figure 9 & Red & $2.442 \leq c \leq 2.488$ & Upward & $(5,0,0,0)$ \\
& Black & $2.488 \leq c \leq 2.442$ & Downward & $(-10.67,0,0,0)$ \\
& Cyan & $2.442 \leq c \leq 2.488$ & Upward & $(12,0,0,0)$ \\
Magenta & $2.442 \leq c \leq 2.488$ & Upward & $(-5.5,0,0,0)$ \\
& Blue & $2.802 \leq m \leq 2.865$ & Upward & $(12.8,0,0,0)$ \\
& Red & $2.738 \leq m \leq 2.802$ & Downward & Upward \\
& & $2.802 \leq m \leq 2.865$ & Downward & $(-10.1,0,0,0)$ \\
\hline
\end{tabular}
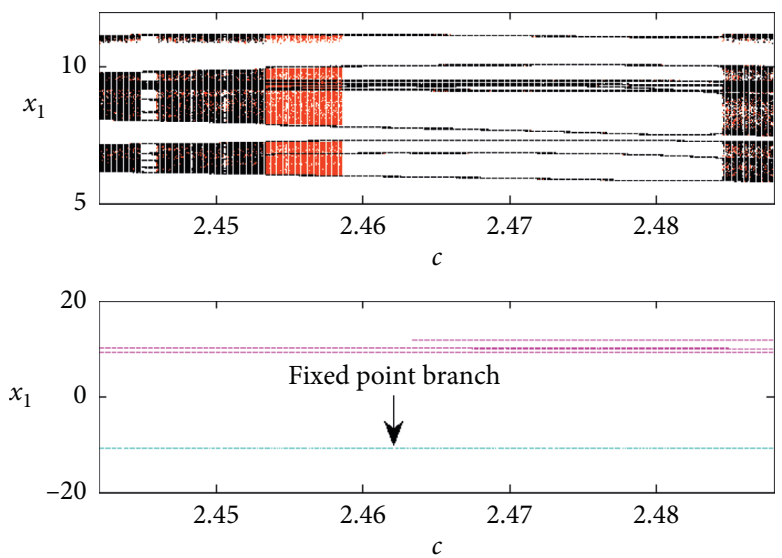

FIGURE 9: Enlargement of bifurcation diagram of Figure 8 plotted in the range $2.442 \leq c \leq 2.488$ showing the region in which the system exhibits multiple coexisting attractors. The system parameters are the same as the one of Figure 8 .

The initial conditions of the coexistence of ten attractors exhibited by the proposed hyperjerk system are presented in Figure 13. The bifurcation like sequence of Figure 13 shows the variation of $x_{3}(0)$ in terms of the control parameter $c$, and the other initial conditions are set to zero $\left(x_{1}(0)=x_{2}(0)=x_{4}(0)=0\right)$. Note that chaotic attractors are characterized by an unlimited number of points, while periodic attractors are characterized by a finite number of points. In the same line, the basin of attraction shows the different domains of convergence of similar attractors. The phase portrait of Figure 14 illustrates the coexistence of ten attractors. The initials conditions of the coexisting attractors are given in Table 4.

3.3.2. Coexistence of Attractors with respect to Bifurcation Parameters $m$ and $b$. In order to investigate the sensitivity of the new hyperjerk system in terms of the bifurcation parameter $m$, the other parameters are fixed as follows: $a=1.8$, $b=3.8, c=2.442, d=1.35$, and $e=14.85$. We found that the novel hyperjerk system can exhibit striking bifurcation sequences when varying the control parameter $m$ in the range $2 \leq m \leq 4.18$. With reference to Figure 11, the bifurcation diagram in black and the one in blue are obtained by increasing and decreasing the values of the parameter $m$, while the one in red is obtained by fixing the initial conditions at $\left(x_{1}(0), x_{2}(0), x_{3}(0), x_{4}(0)\right)=(-4,0,0,0)$. A window of hysteretic dynamics can be identified in the range $2.6 \leq m \leq 2$.9. The enlarged bifurcation diagram of Figure 12 clearly illustrates the domain of the coexistence of multiple attractors observed in the new hyperjerk system according to the bifurcation parameter $m$. Different methods used to plot these bifurcation diagrams are presented in Table 3. Up to six different periodic, chaotic, and point attractors can be obtained by only changing the initial conditions. For instance, sample phase portraits of the coexistence of six distinct attractors are presented in Figure 13.

During the mathematical analyses, it has been shown that the Hopf bifurcation was depending on the control parameter $m$. The bifurcation diagram of Figure 13(a) clearly illustrates this 


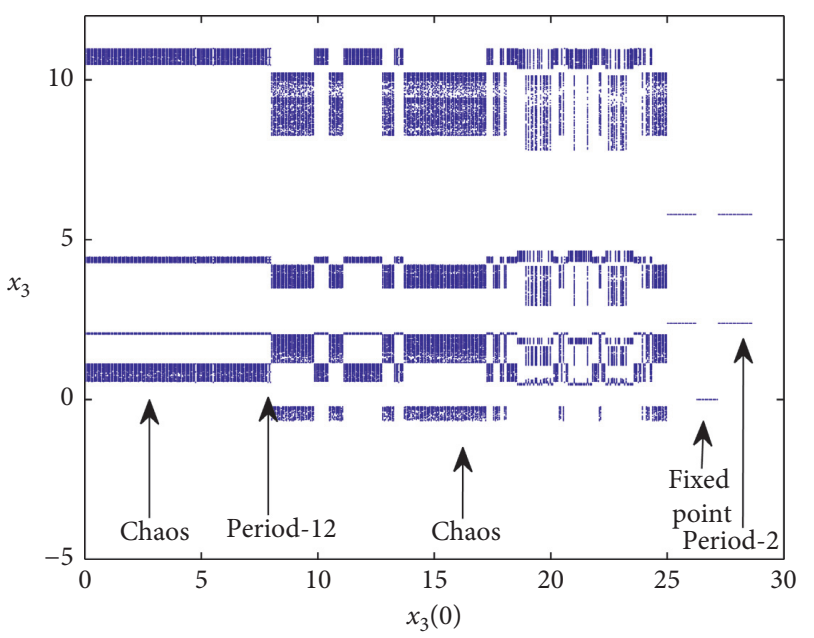

FIGURE 10: Bifurcation like sequence showing local maxima of the coordinate $x_{3}$ versus the initial condition. plotted in the range $0 \leq x(0) \leq 30$, with $a=1.8, b=3.8, c=2.5442, d=1.35$, and $e=14.85$. It can be observed the coexistence of ten periodic, chaotic, and point attractors.

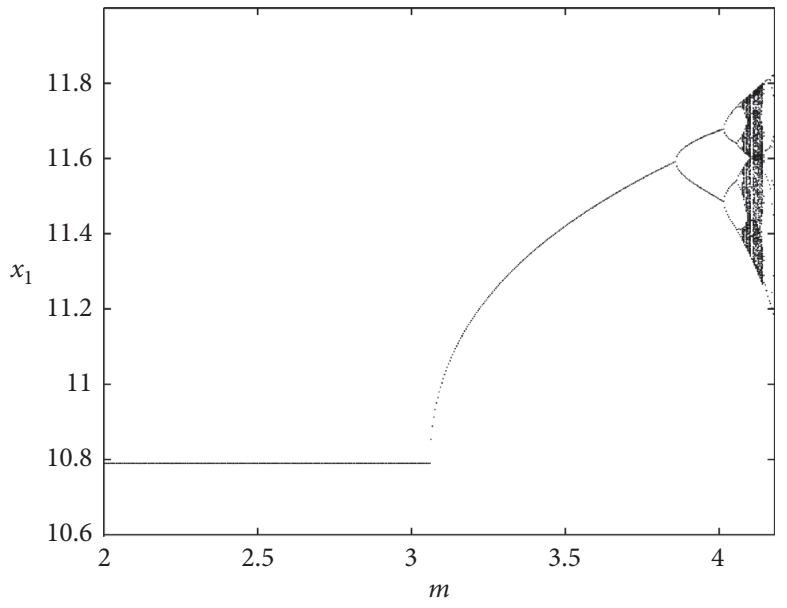

(a)

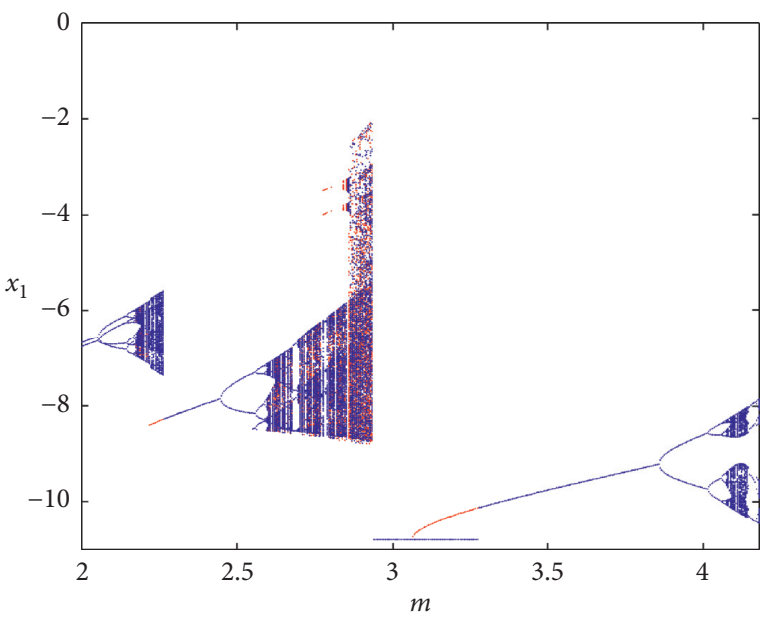

(b)

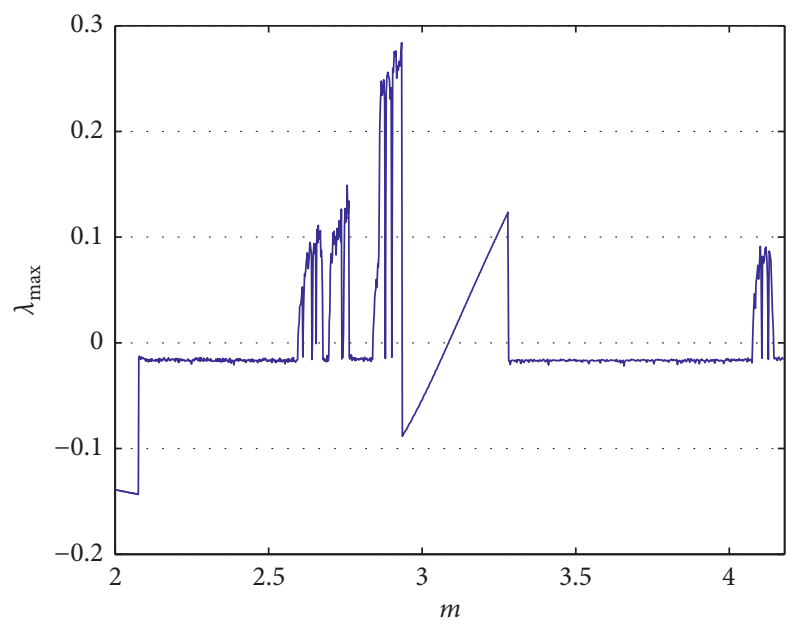

(c)

Figure 11: $(\mathrm{a}, \mathrm{b})$ Bifurcation diagrams showing local maxima of the coordinate $x_{1}$ versus $m$ and (c) the corresponding graph of largest Lyapunov exponent $\left(\lambda_{\max }\right)$ plotted in the range $2 \leq m \leq 4.18$, with $a=1.8, b=3.8, c=2.442$, and $d=1.35$. 


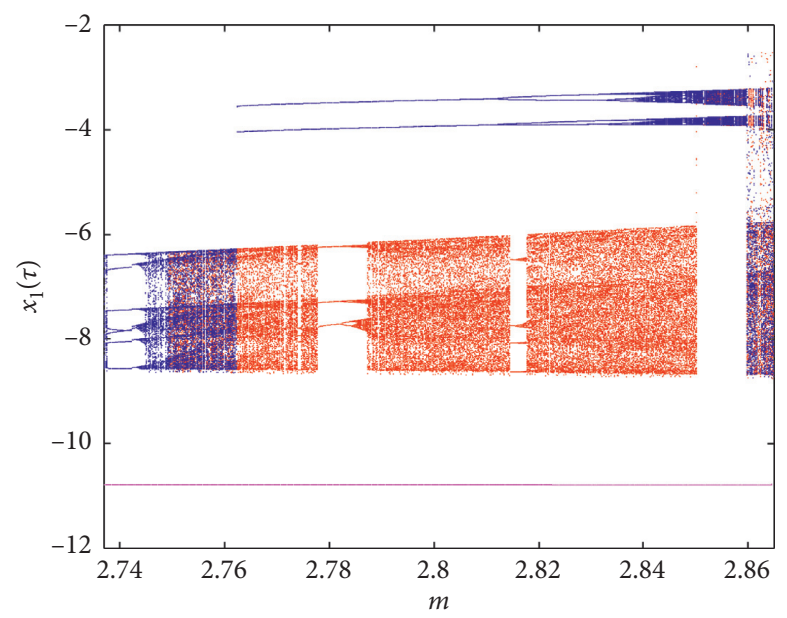

FIGURE 12: Enlargement of the bifurcation diagram of Figure 11(a) showing the region in which the system exhibits multiple coexisting attractors. This region corresponds to values of $m$ in the range: $2.735 \leq m \leq 2.865$. Three sets of data are superimposed. For more information about the methods used to plot, see Table 3.

phenomenon characterized by the stable state followed by the unstable state. Moreover, this control parameter also highlights the coexistence of multiple attractors exhibited by the new 4-D system. By considering the following sets of the parameters: $a=1.8, b=3.5, c=2.442, d=1.35, e=15$, and $m=3$, we discover that the new 4-D system displays the coexistence of four distinct chaotic and point attractors. The phase portraits of the Figure 14 and their corresponding cross section of the basin of attraction clearly show the coexistence phenomenon and also give the initial condition domain of each attractor. The green and black domains represent the initial conditions regions of the pair of chaotic attractors, while the yellow domain represents the initial condition regions of the pair of point attractors.

3.4. Offset Boosting Scenario. Another property of system (3) is the possibility to develop an offset boosting effect. In our model, $x_{1}$ appears only in the fourth line of equation (3), and thus this variable is a bootable variable [47-52]. Assuming the transformation $x_{1} \longrightarrow x_{1}+k$ where $k$ is a constant, equation (5) can be rewritten accordingly as

$$
\left\{\begin{array}{l}
\dot{x}_{1}=x_{2}, \\
\dot{x}_{2}=m x_{3}, \\
\dot{x}_{3}=d x_{4}, \\
\dot{x}_{4}=c\left(x_{1}+k\right)-b x_{2}-e x_{3}-a x_{4}-\gamma \sinh \left(x_{1}+k\right) .
\end{array}\right.
$$

Figure 19 clearly presents offset boosting of the doubleband chaotic attractor. The following values of parameter: $k=0$ (blue), $k=10$ (red) and $k=-10$ (green) are used to plot them in $x_{1}-x_{4}$ and $x_{1}-x_{3}$ planes.

3.5. Antimonotonicity. By decreasing the value of the control parameter $e(15 \leq e \leq 20)$, we can observe the formation and destruction of periodic orbits via reverse period-doubling bifurcation sequences. This interesting phenomenon has been reported in the literature. It is reported in various nonlinear systems such as Duffing oscillator [12], Chua circuit [53], and second-order nonlinear nonautonomous circuit $[54,55]$. This phenomenon was reported for the first time in the hyperjerk system by Leutcho et al. [21]. The creation of periodic seas in the parameter space is the necessary requirement for a nonlinear system to experience forward and reverse perioddoubling cascade [21]. Sample illustrations are represented in Figure 20, where some bifurcation diagrams are shown. These diagrams are obtained for each discrete value of the control parameter $c$. In Figure 20, note that for $c=2.5442$, we have period-2 bubble and for a slight adjustment of the control parameter $c$, period-4 bubble is observed for $c=2.8442$, whereas for $c=2.9$, we have a period- 8 bubble. In the same order, chaotic bubbles are formed for $c=2.97$, and $c=2.99$. The increase of control parameter $c$ causes the creation of other bubbles, and it finally results in an infinite tree (like chaos).

\section{Experimental Study}

The objective of this section is to confirm the above theoretical results by realizing a laboratory experimental study. For this purpose to be achieved, several approaches have been proposed in the literature to implement chaotic circuit (by using many types of off-the-shelf electronic components [56] or field-programmable gate array (FPGA) technology [57-60] or field-programmable-analog-array (FPAA) technology $[61,62]$ just to name a few). Only off-the-shelf electronic components (i.e., resistors, capacitors, pair of semiconductor diodes $\left(D_{1}=D_{2}=1 N 4148\right)$, and TL084 operational amplifiers types with a power supply of \pm 15 VDC) are used to realize 


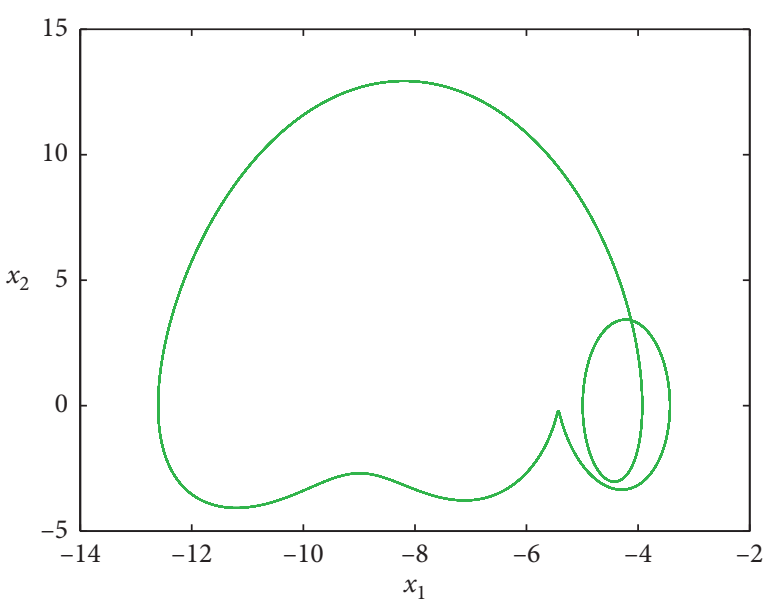

(a)

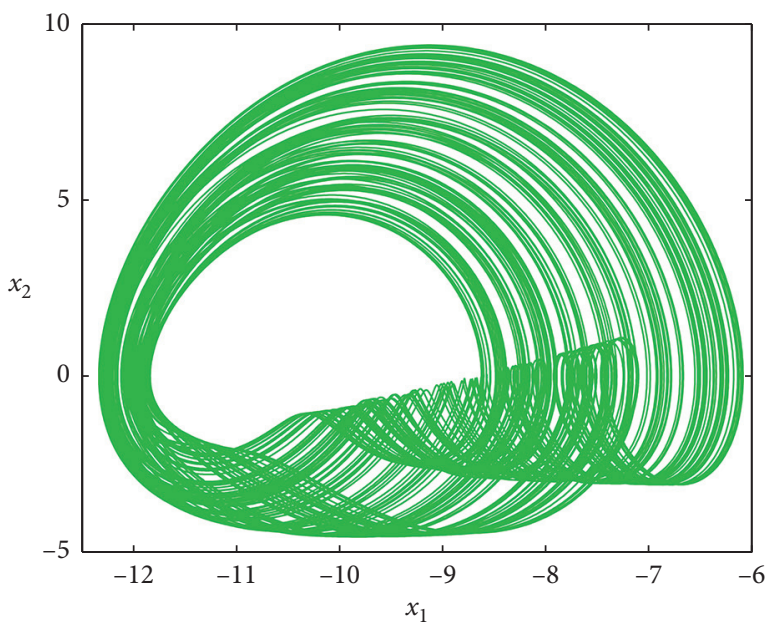

(c)

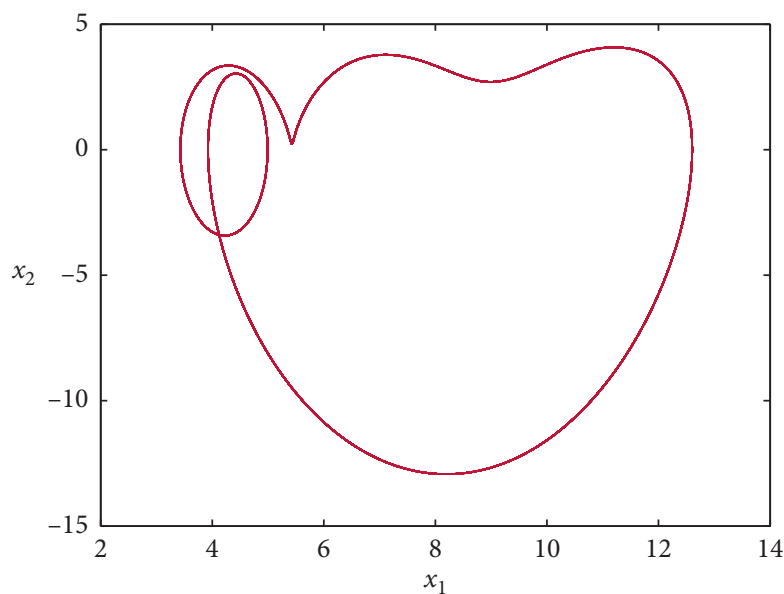

(b)

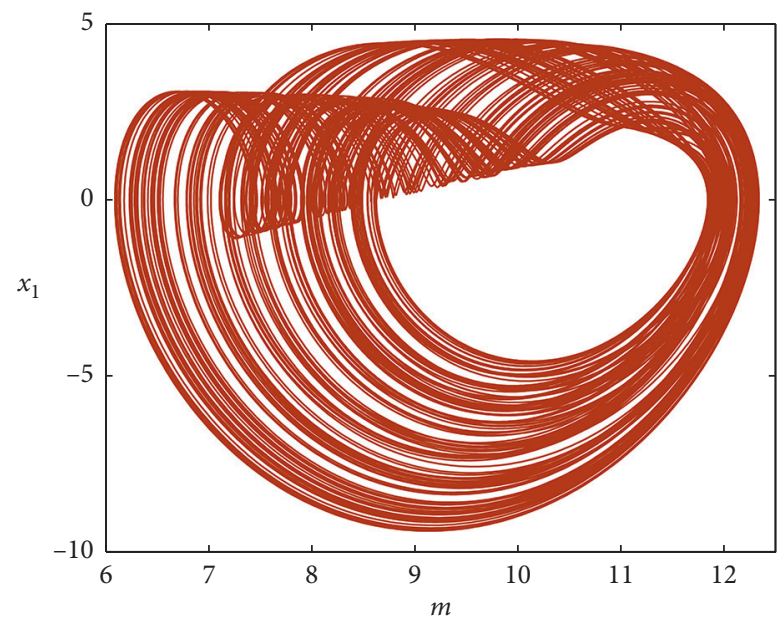

(d)

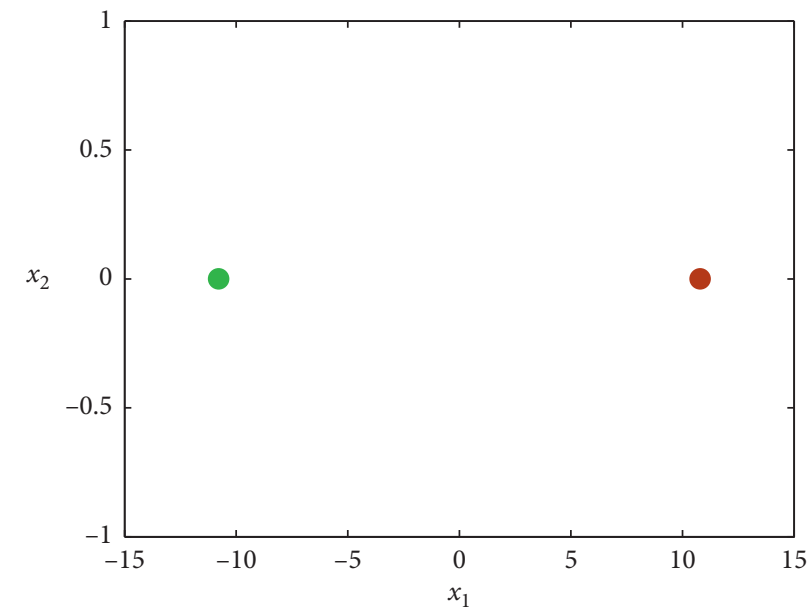

(e)

FIGURE 13: Coexistence of six different attractors (a pair of period-2 limit cycle, a pair of chaotic attractors, and a pair of fixed point attractor) for the following values of system parameters: $a=1.8, b=3.8, c=2.442, d=1.35, e=14.85$, and $m=2.802$. Initial conditions $\left(x_{1}(0), x_{2}(0), x_{3}(0), x_{4}(0)\right)$ are, respectively, $( \pm 5.5,0,0,0),( \pm 12.8,0,0,0)$, and $( \pm 10.1,0,0,0)$.

the schematic diagram of Figure 1. The following values of electronic circuit components are used during the experimental process: $R_{c}=R_{m}=R=10 \mathrm{k} \Omega, R_{b}=1.67 \mathrm{k} \Omega$,
$R_{e}=5 k \Omega$, and $R_{d}=2.941 k \Omega$ (for the other parameters, see Table 1 case A). The complete sequence of phase portraits plotted in $\left(x_{1}, x_{4}\right)$ plan is obtained by adjusting 


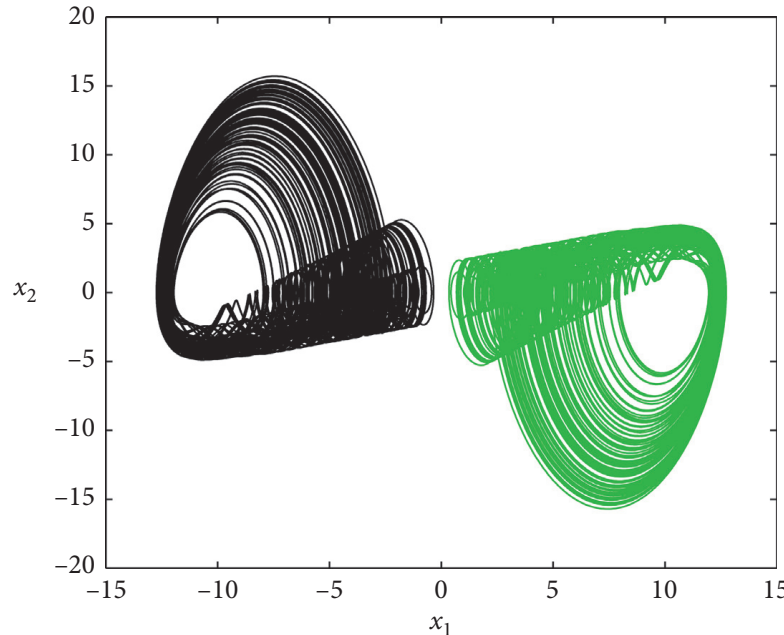

(a)

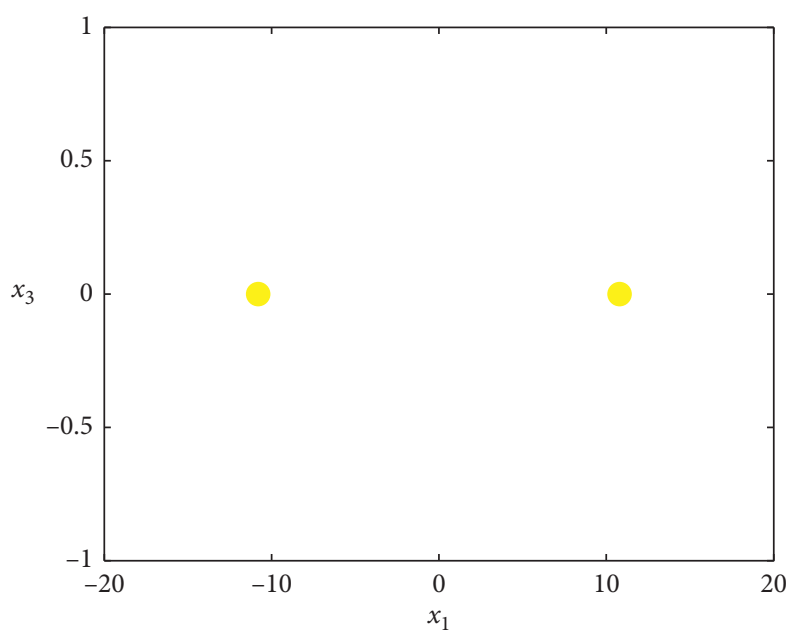

(c)

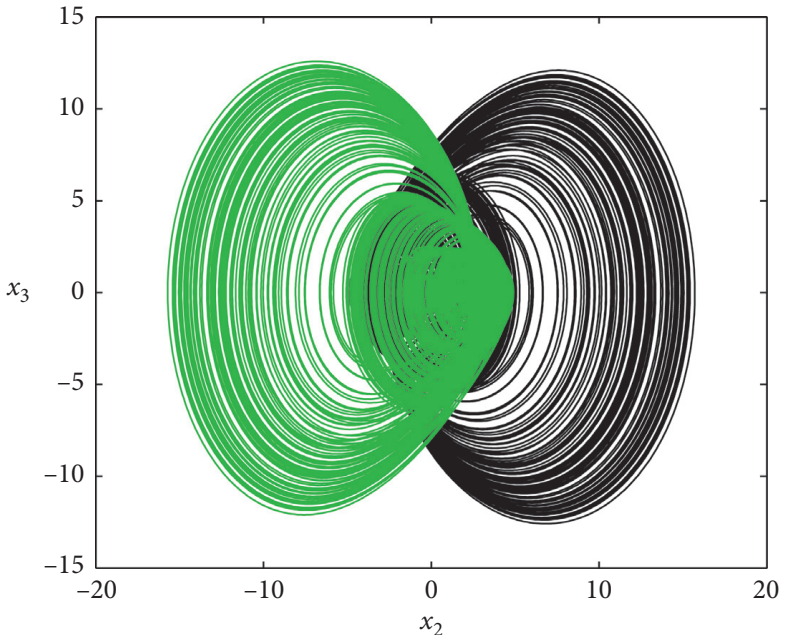

(b)

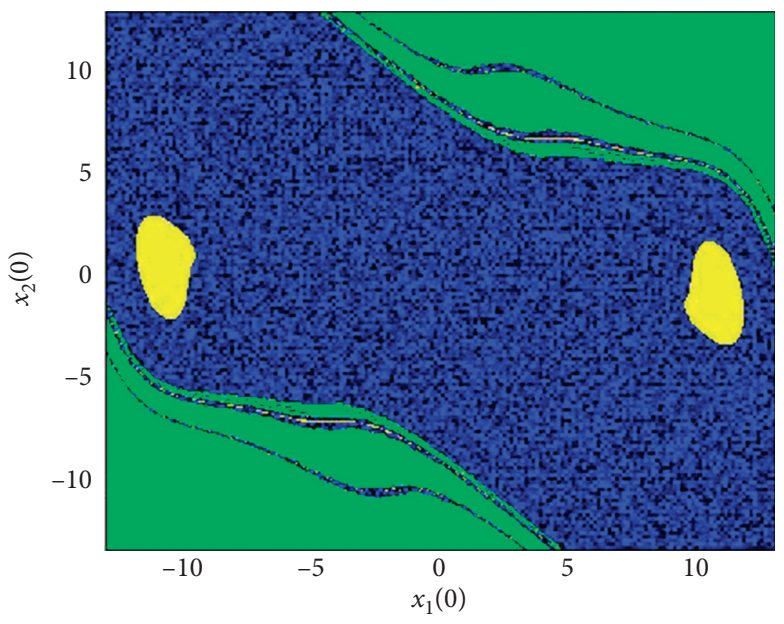

(d)

Figure 14: (a, b) Coexistence of four distinct attractors (a pair of chaotic attractors represented in $x_{1}-x_{2}$ and $x_{2}-x_{3}$ plans), (c) a pair of fixed point attractor) and their corresponding basin of attraction. Initial conditions $\left(x_{1}(0), x_{2}(0), x_{3}(0), x_{4}(0)\right)$ are, respectively, $( \pm 7.15,0, \pm 8.613,0)$ and $( \pm 12.8,0,0,0)$. The rest of the parameters are $a=1.8, b=3.5, c=2.442, d=1.35, e=15$, and $m=3$.

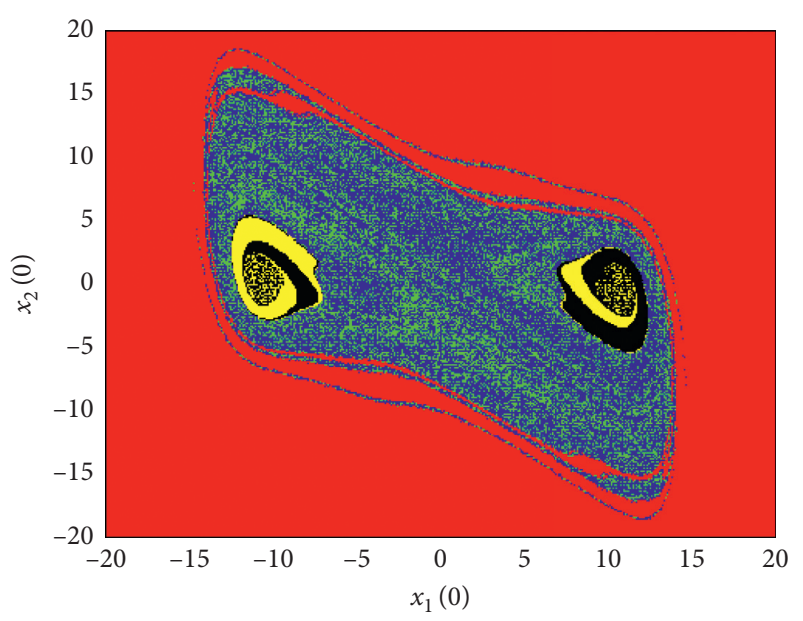

(a)

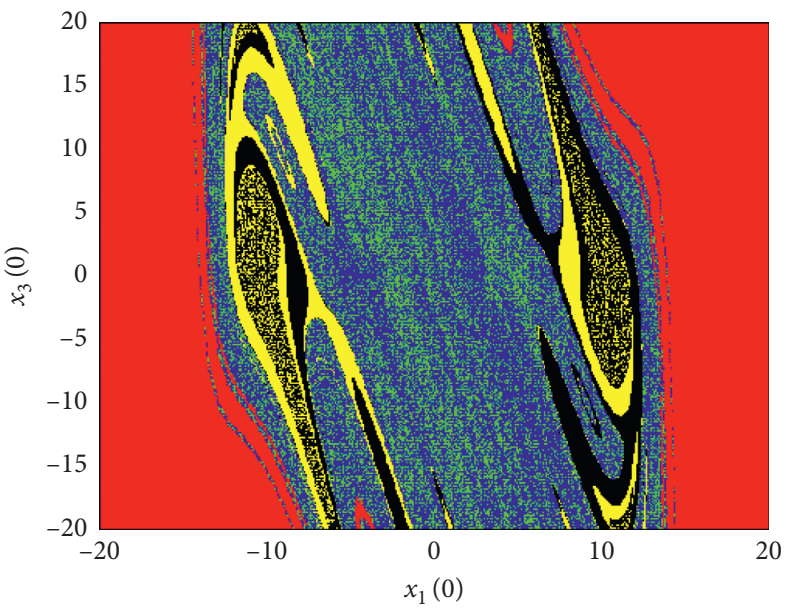

(b)

Figure 15: Continued. 


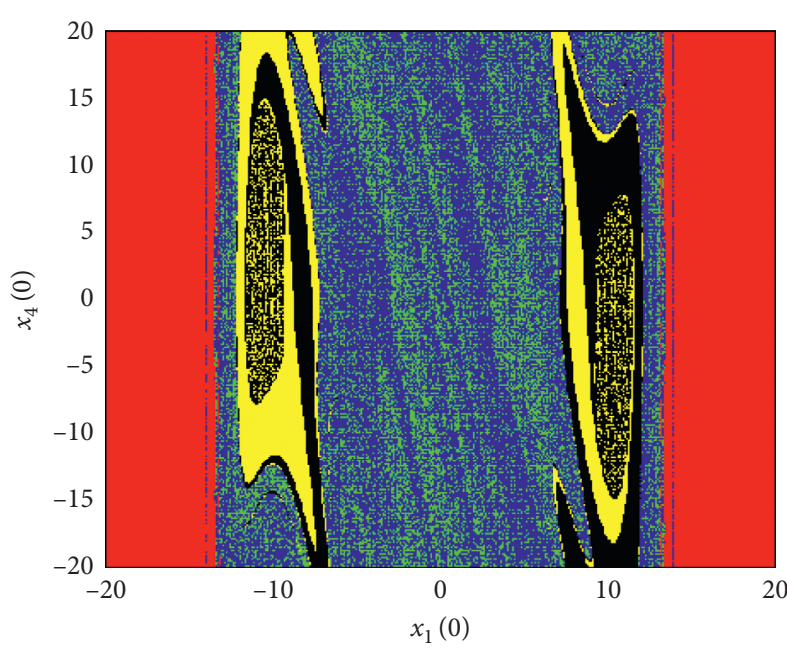

(c)

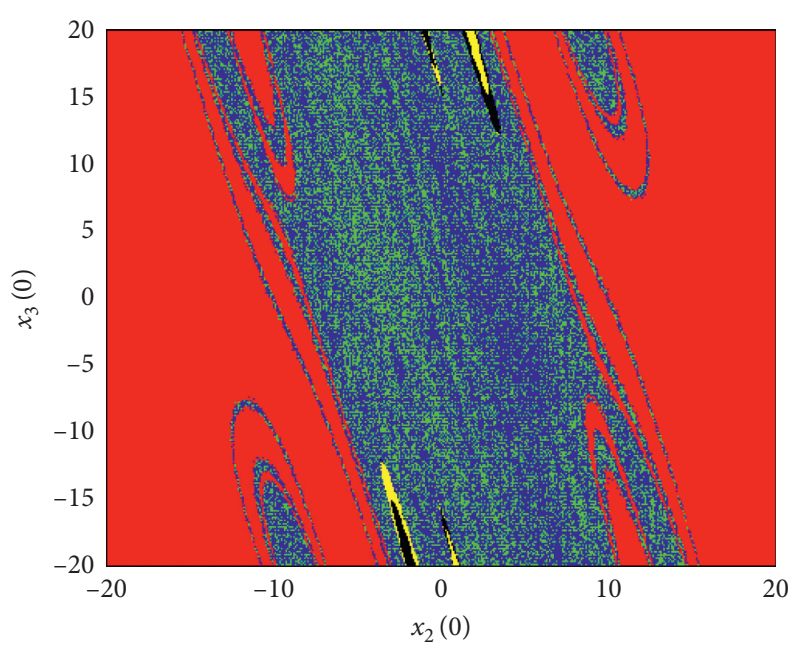

(d)

FIgURE 15: Cross sections of basin of attraction for $x_{3}(0)=x_{4}(0)=0, x_{2}(0)=x_{4}(0)=0, x_{2}(0)=x_{3}(0)=0$, and $x_{1}(0)=x_{4}(0)=0$ corresponding to the asymmetric pair of chaotic (blue and green) and period-2 attractors (yellow and black) obtained for $c=2.44$. Red regions correspond to unbounded motion.

TABLE 4: Details of the coexistences observed in the novel hyperjerk system.

\begin{tabular}{|c|c|c|c|}
\hline Fig. $n^{0}$ & Type of coexistences & $\begin{array}{l}\text { Values of control } \\
\text { parameter }\end{array}$ & $\begin{array}{c}\text { Initial condition } \\
\left(x_{1}(0), x_{2}(0), x_{3}(0), x_{4}(0)\right) \\
\end{array}$ \\
\hline Figure 16 & $\begin{array}{l}\text { A symmetric pair of period-2 attractors, a symmetric pair of chaotic } \\
\text { attractors, and a pair of fixed point. }\end{array}$ & $c=2.44$ & $\begin{array}{l}\text { (a) }(0,0, \pm 44.4,0) \\
\text { (b) }(0,0, \pm 48,0) \\
\text { (c) }(0,0, \pm 26.4,0)\end{array}$ \\
\hline Figure 17 & $\begin{array}{c}\text { A symmetric pair of period- } 2 \text { attractors, a symmetric pair of chaotic } \\
\text { attractors, a symmetric pair of period- } 9 \text { attractors, and a pair of fixed } \\
\text { point. }\end{array}$ & $c=2.454$ & $\begin{array}{l}\text { (a) }(0,0, \pm 25.2,0) \\
\text { (b) }(0,0, \pm 48,0) \\
\text { (c) }(0,0, \pm 10.8,0) \\
\text { (d) }(0,0, \pm 26.4,0)\end{array}$ \\
\hline Figure 18 & $\begin{array}{l}\text { A symmetric pair of period-2 attractors, a fixed point, a symmetric pair of } \\
\text { period-12 attractors, } 2 \text { symmetric pairs of chaotic attractors, and a pair of } \\
\text { fixed point. }\end{array}$ & $c=2.5442$ & $\begin{array}{c}\left(\mathrm{A}^{\prime \prime} A_{1}, A_{2}{ }^{\prime \prime}\right)(0,0, \pm 45.6,0) \\
\text { (b) }(0,0, \pm 18,0) \\
\text { (c) }(0,0, \pm 49.2,0) \\
\text { (d) }(0,0, \pm 48,0) \\
\text { (e) }(0,0, \pm 26.4,0)\end{array}$ \\
\hline
\end{tabular}

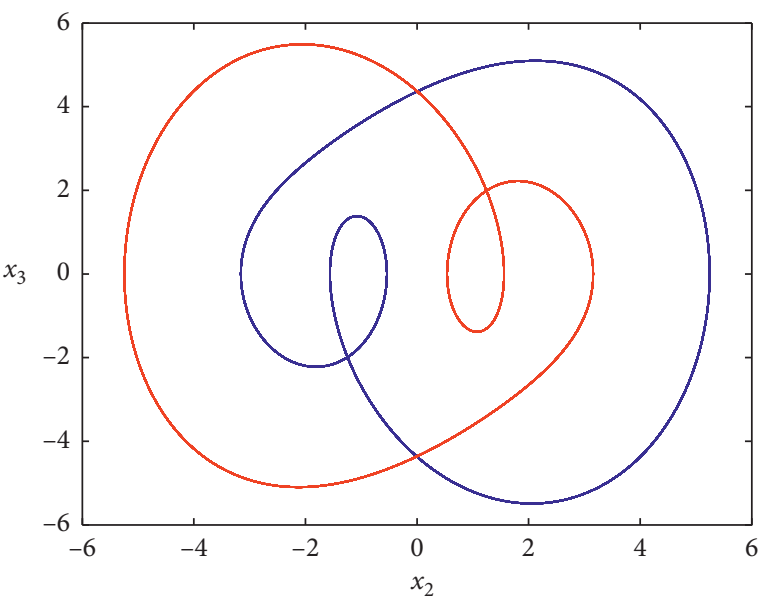

(a)

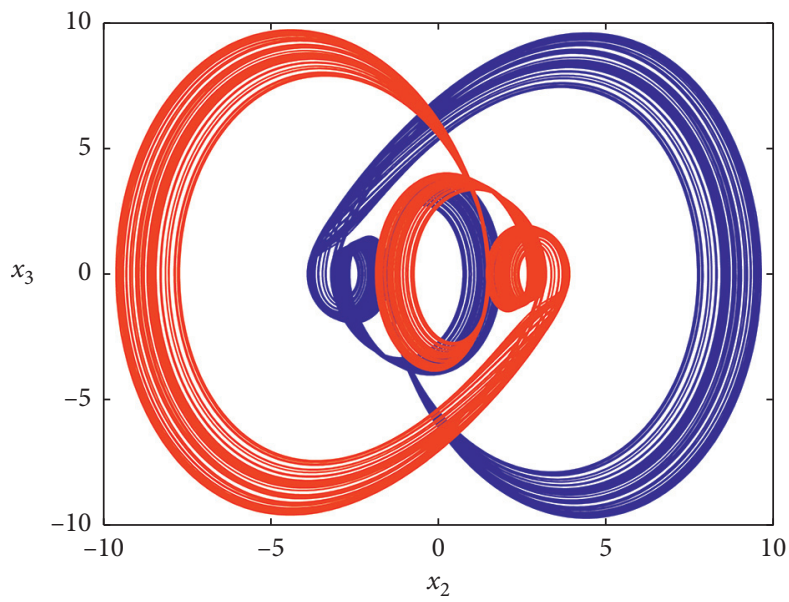

(b)

Figure 16: Continued. 


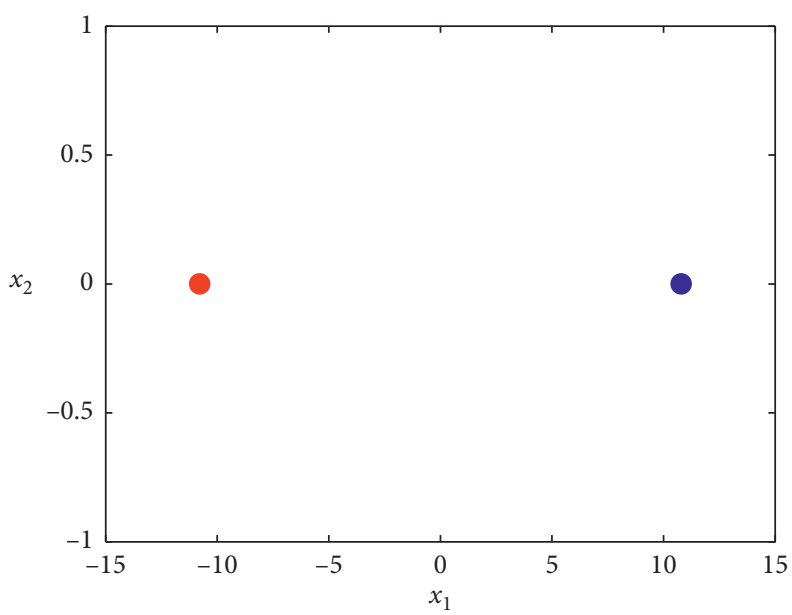

(c)

Figure 16: Coexistence of six different attractors (a pair of symmetric period-2 limit cycle, a pair of symmetric period-9 limit cycle, a pair of symmetric chaotic attractors, and a pair of point attractors). The values of others system parameters are fixed as follows: $a=1.8, b=3.8$, $d=1.35, e=14.85$, and $m=2.2$. Initial conditions are given in Table 4 .

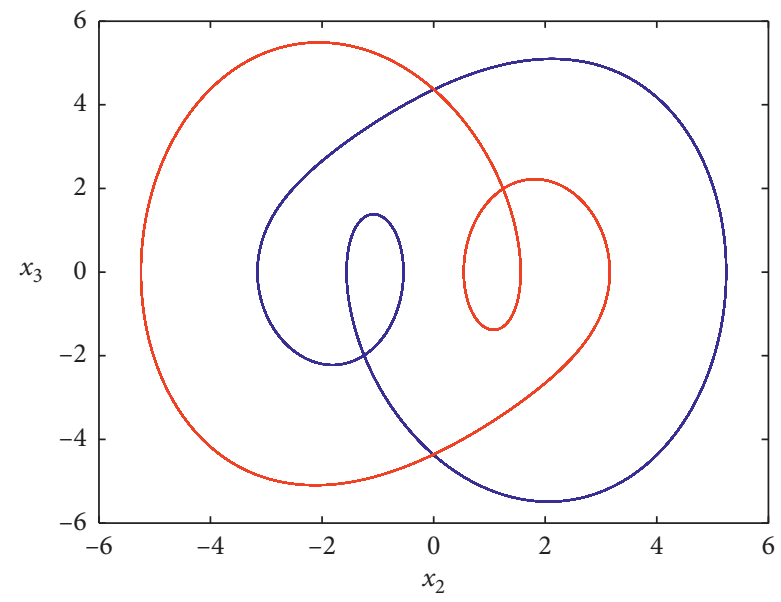

(a)

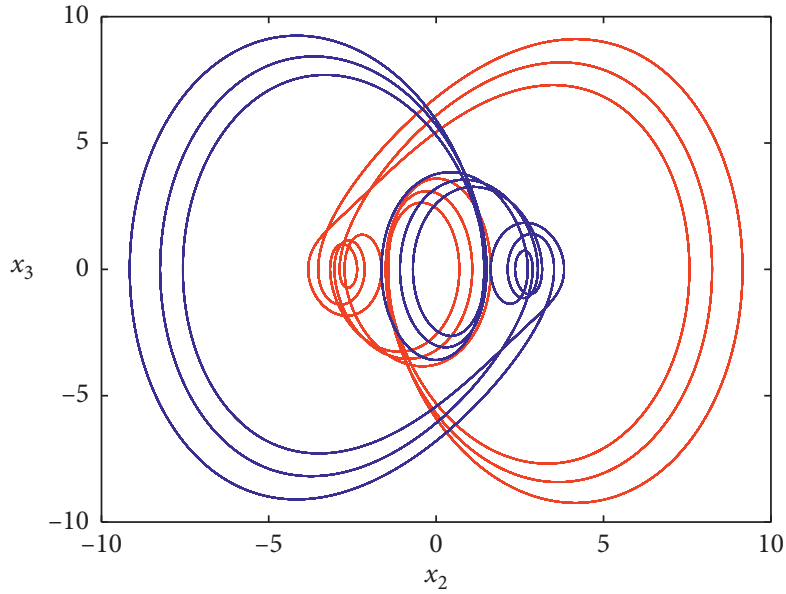

(c)

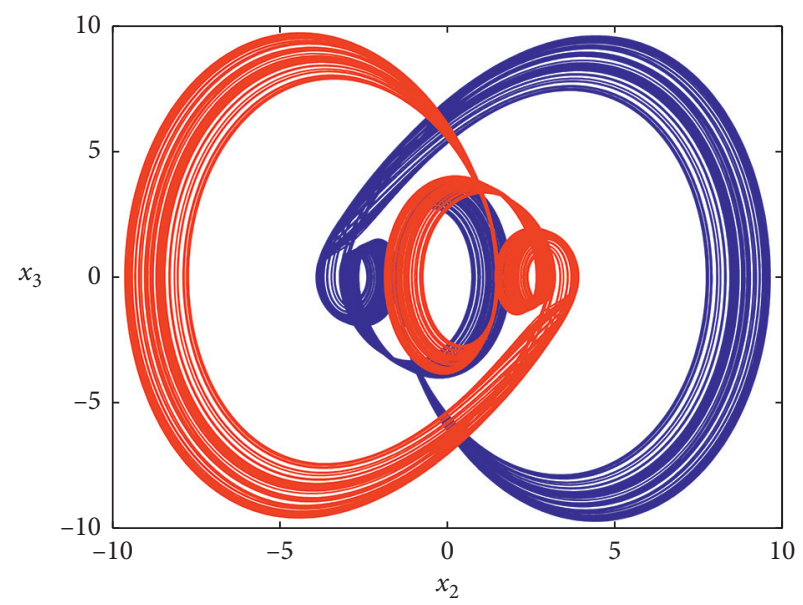

(b)

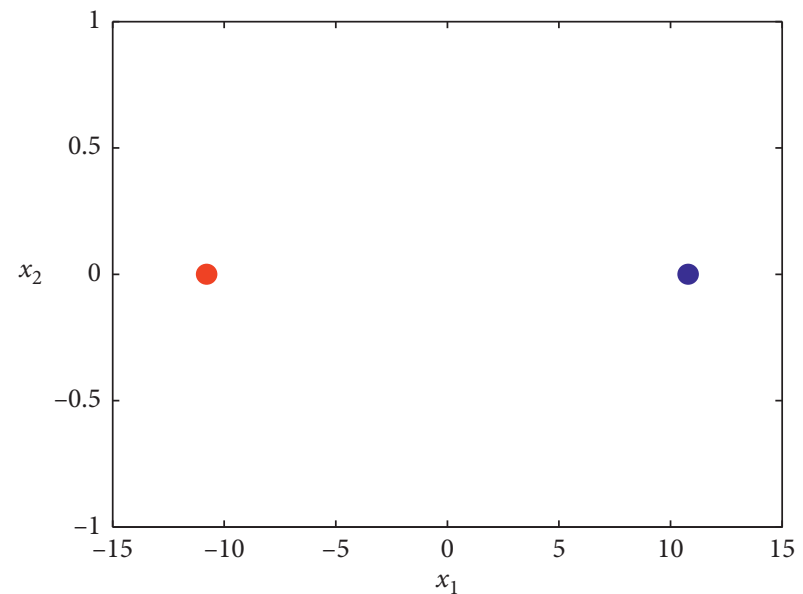

(d)

FIGURE 17: Coexistence of eight different attractors (a pair of symmetric period-2 limit cycle, a pair of symmetric chaotic attractors, and a pair of point attractors). The parameters are the same as those in Figure 16. Initial conditions are given in Table 4. 


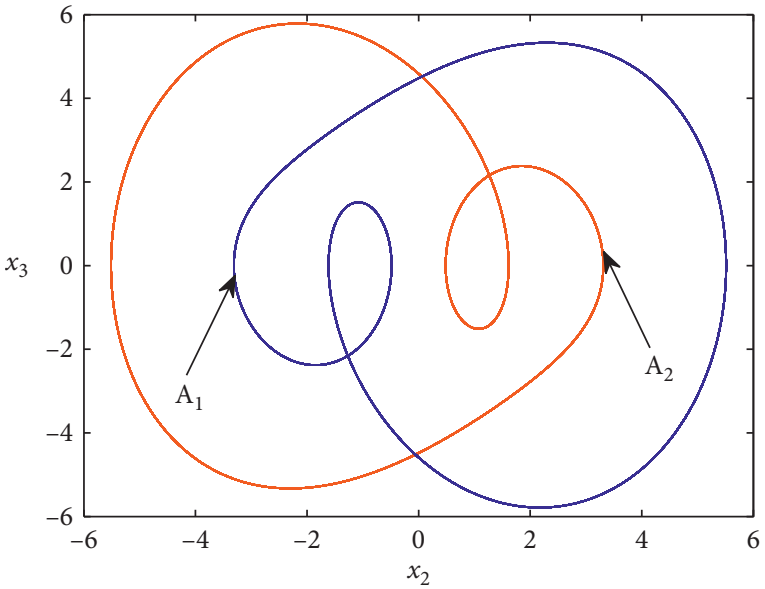

(a)

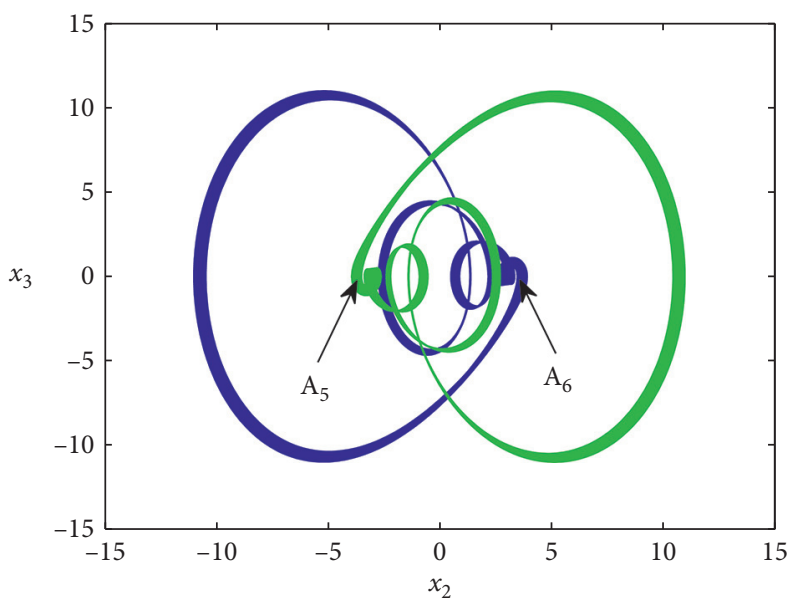

(c)

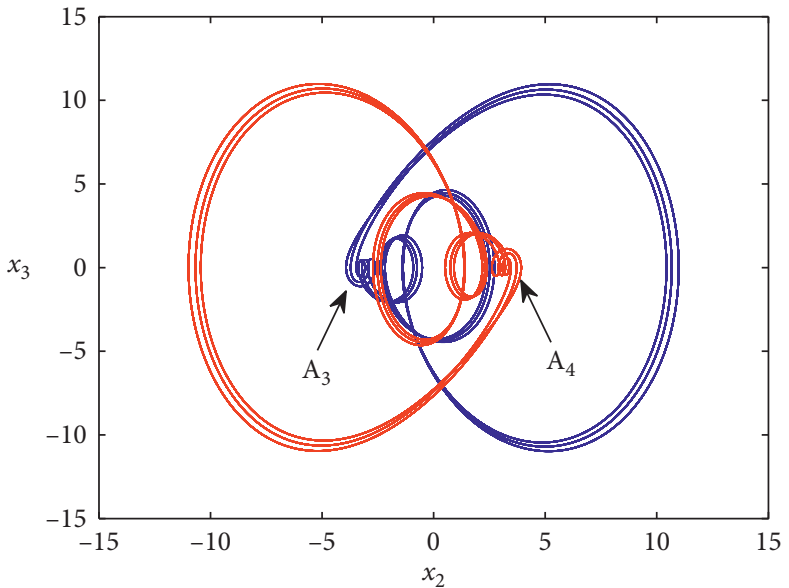

(b)

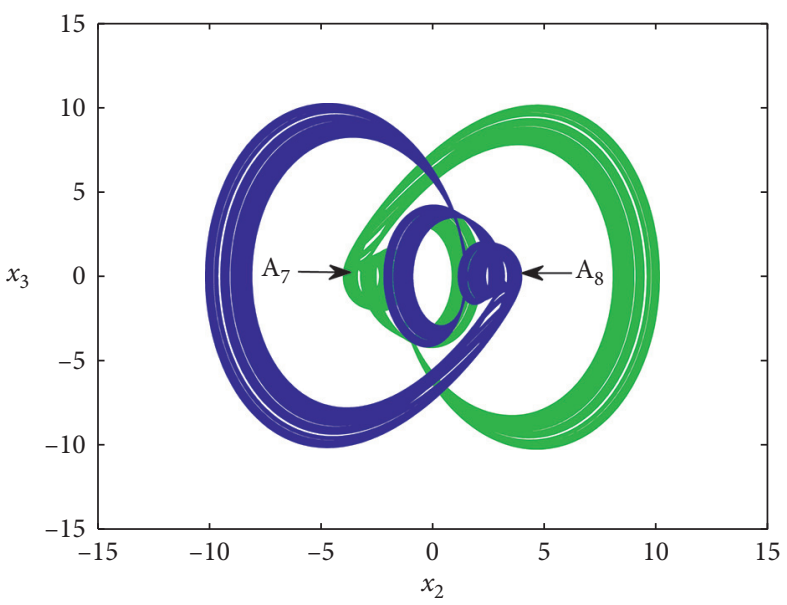

(d)

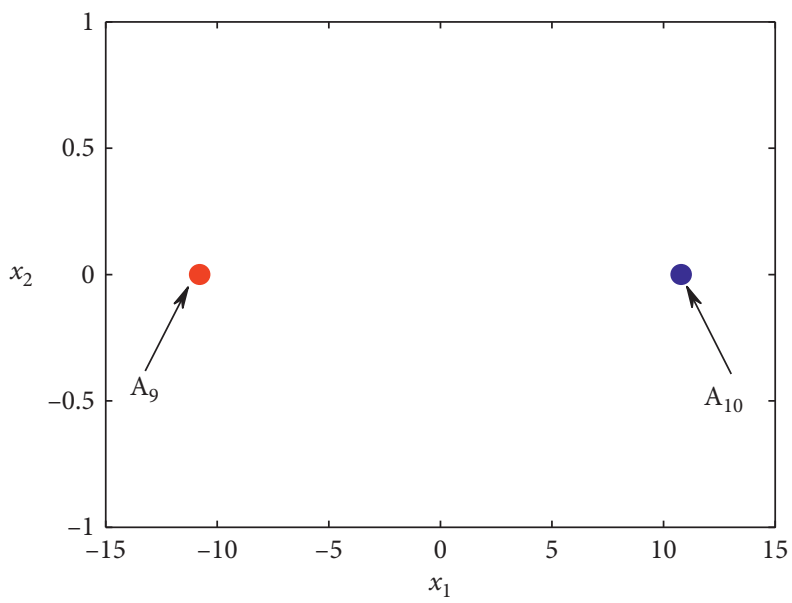

(e)

Figure 18: Coexistence of ten different attractors (a pair of symmetric period-2 limit cycle, 2 pair of symmetric chaotic attractors, a pair of symmetric period-9 limit cycle, and a pair of point attractors). The parameters are the same as those in Figure 16. Initial conditions are given in Table 4.

the control resistor $R_{a}$ in the range $333,33 \Omega \leq R_{a} \leq 1.66 \mathrm{k} \Omega$. It can be seen in Figure 21 a good coherence between the numerical results (left side) and the experimental ones (right side). By changing the values of electronic components: $R_{e}=600 \Omega, R_{a}=2.116 \mathrm{k} \Omega, R_{b}=$ $4.21 \mathrm{k} \Omega, R_{m}=784 \Omega, R_{d}=3.234 \mathrm{k} \Omega$, and $R_{c}=5.16 \mathrm{k} \Omega$ (for the other parameters, see Table 1 case $B)$, the coexistence of attractors emerges. Figure 22 clearly illustrates the 


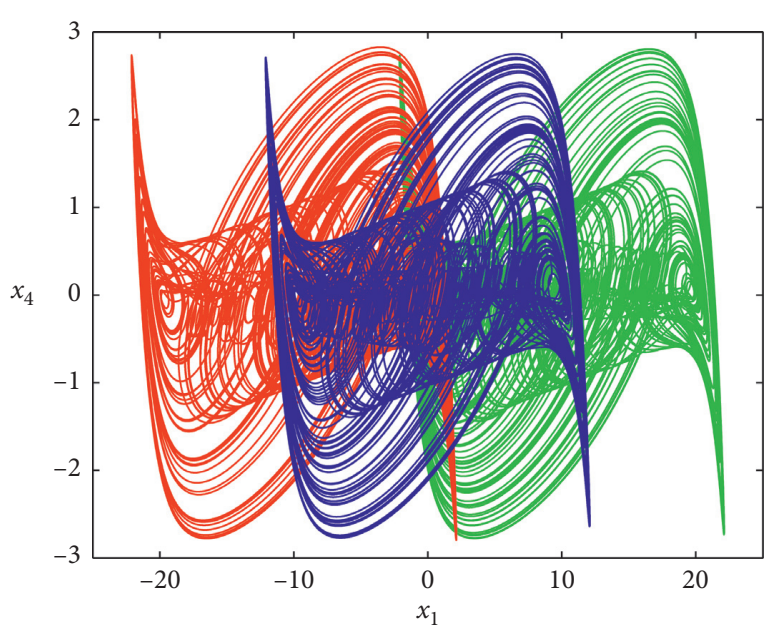

(a)

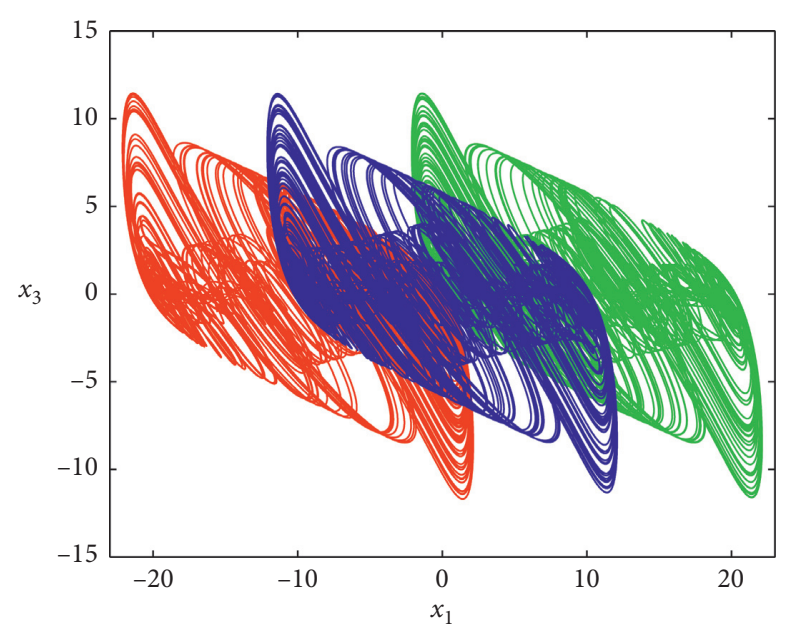

(b)

Figure 19: Offset boosting of the double-band chaotic attractor with the following values of parameter: $k=0$ (blue), $k=10$ (red), and $k=-10$ (green). The other parameters are the same as those in Figure 4.

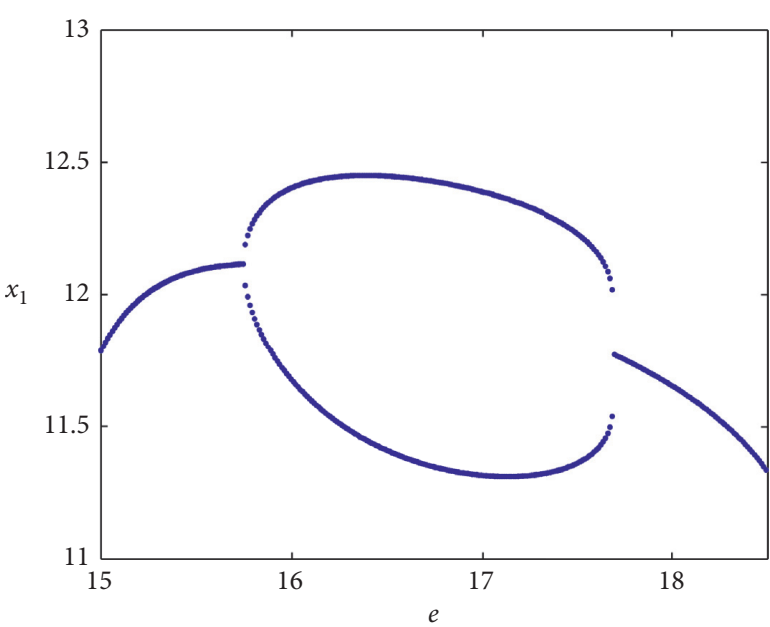

(a)

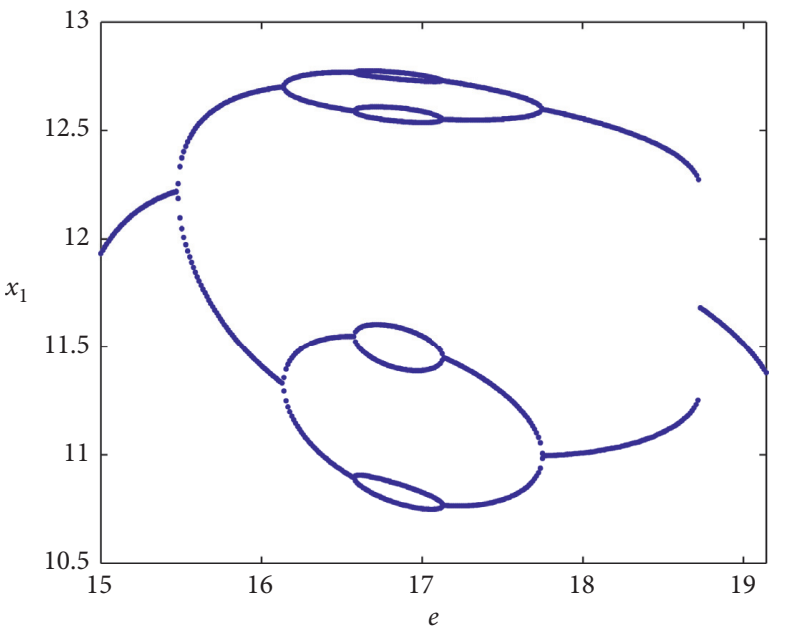

(c)

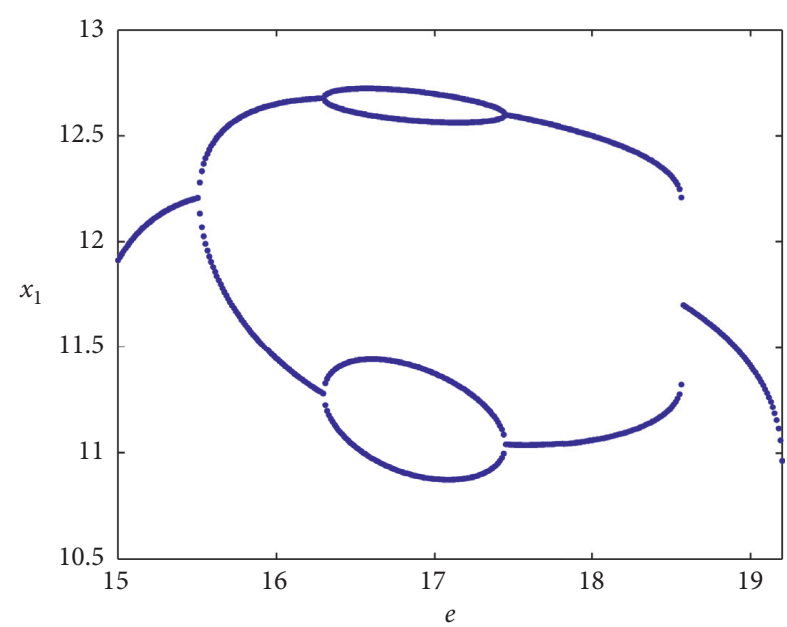

(b)

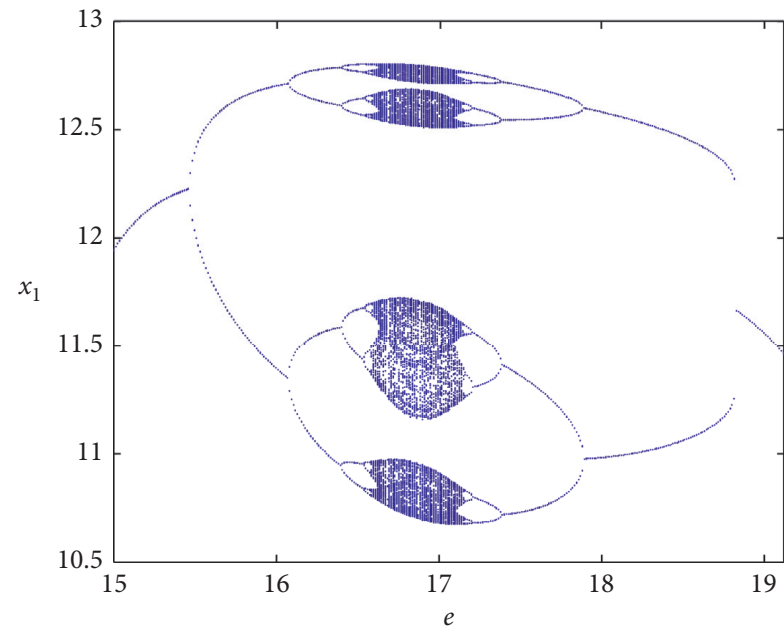

(d)

Figure 20: Continued. 


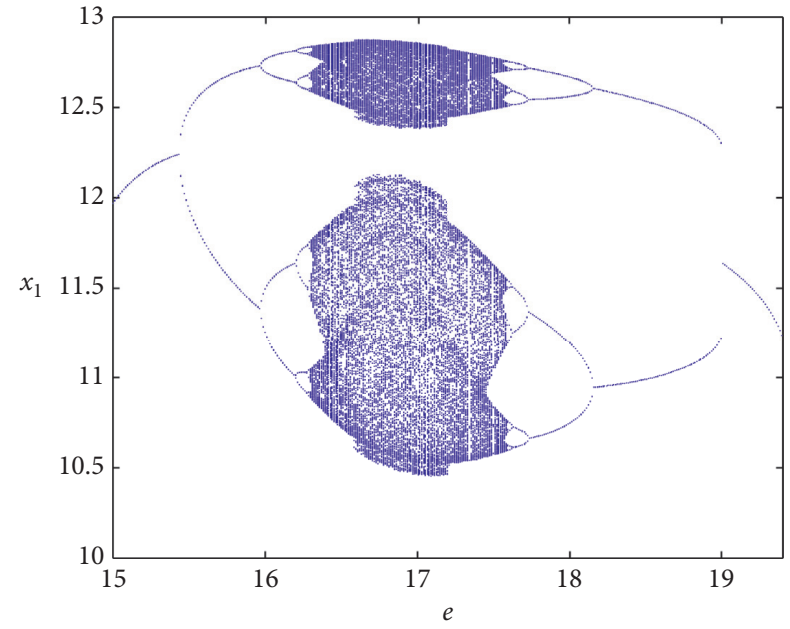

(e)

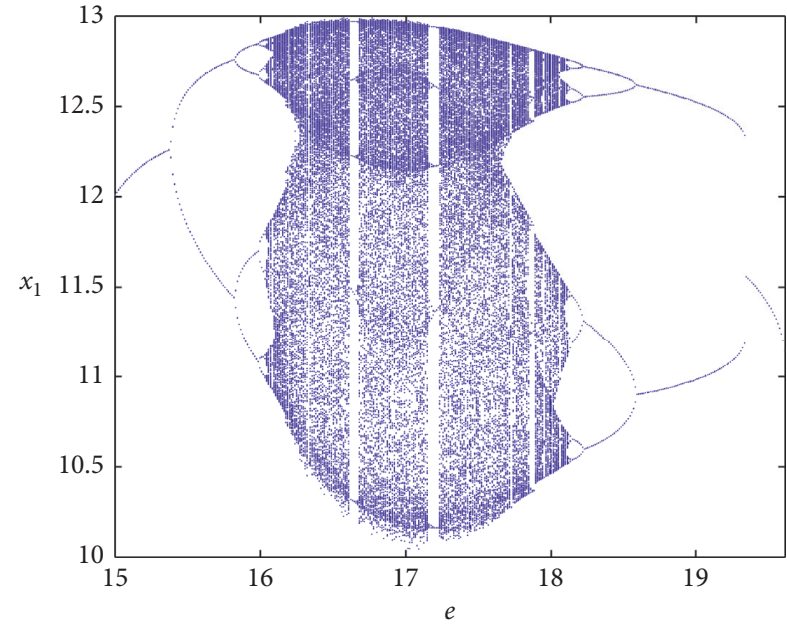

(f)

Figure 20: Bifurcation diagrams showing local maxima of the coordinate $x_{1}$ of the attractor in Poincare cross section in terms of the control parameter $a$ (bubbling): (a) period-2 bubble for $c=2.5442$, (b) period-4 bubble for $c=2.8442$, (c) period-8 bubble for $c=2.9$, (d) singleband chaos bubble for $c=2.93$, (e) single-band chaos bubble for $c=2.99$, and (f) double-band chaos bubble for $c=3.0$.
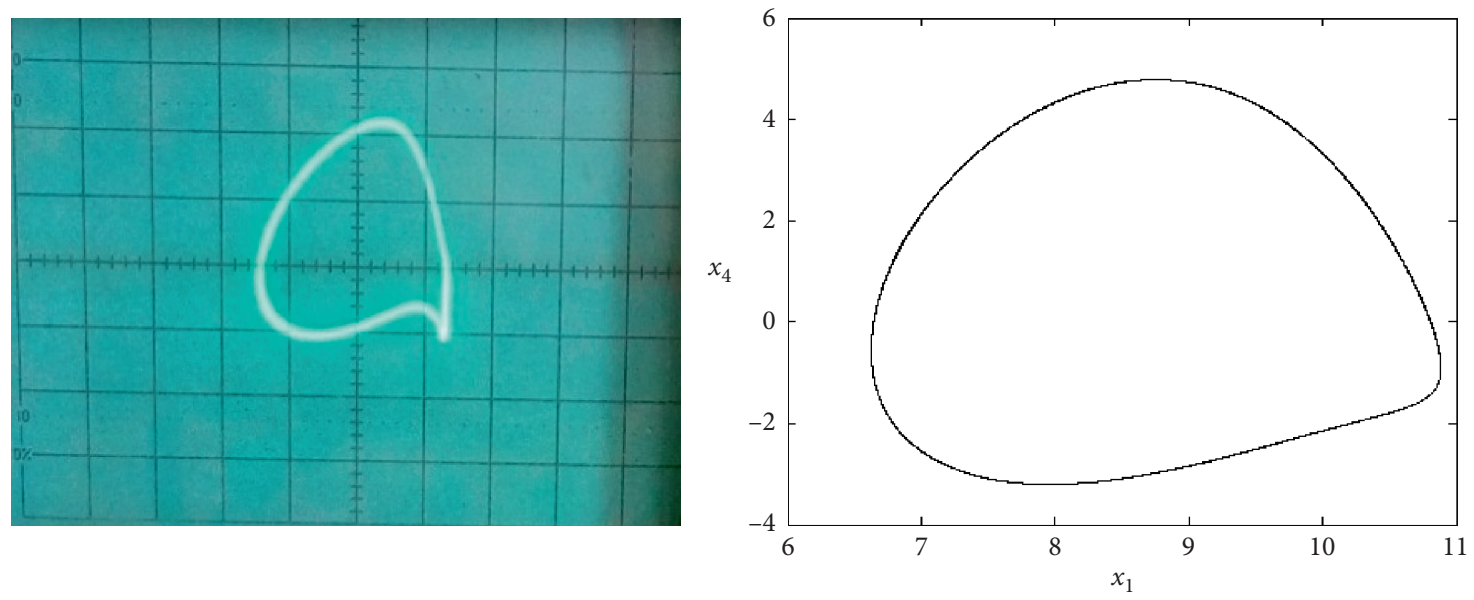

(a)
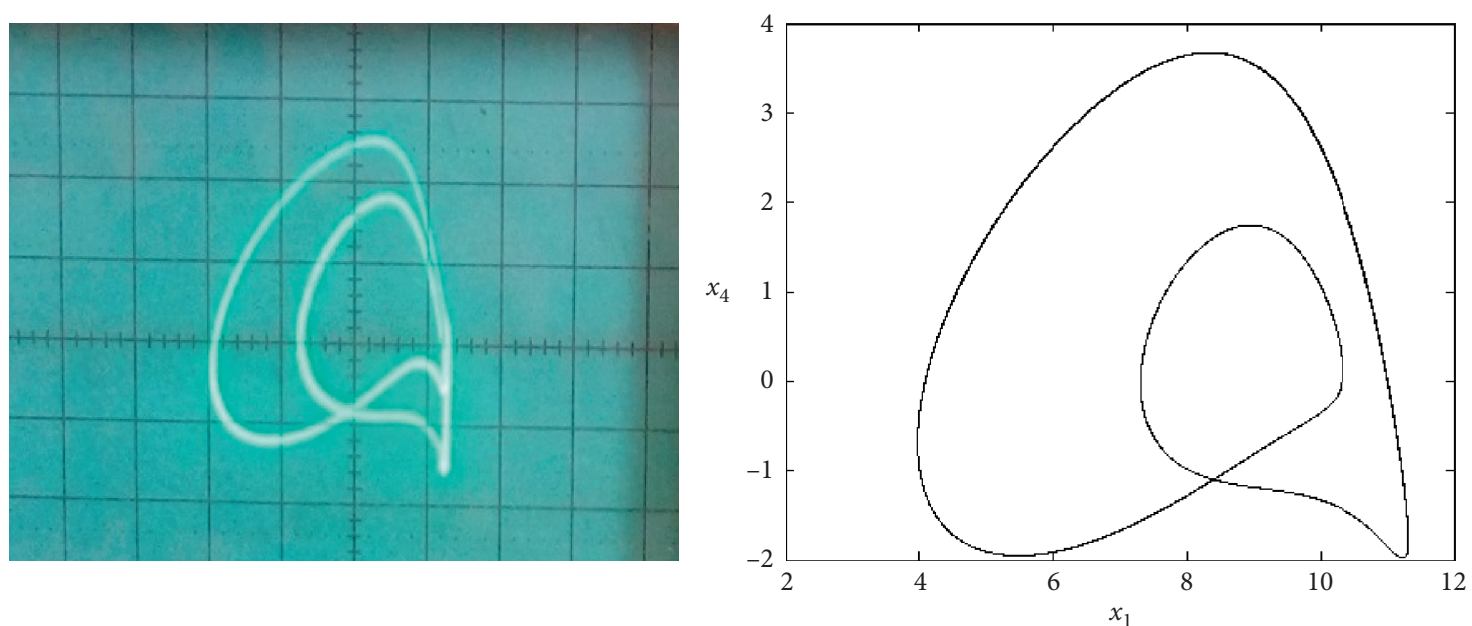

(b)

Figure 21: Continued. 

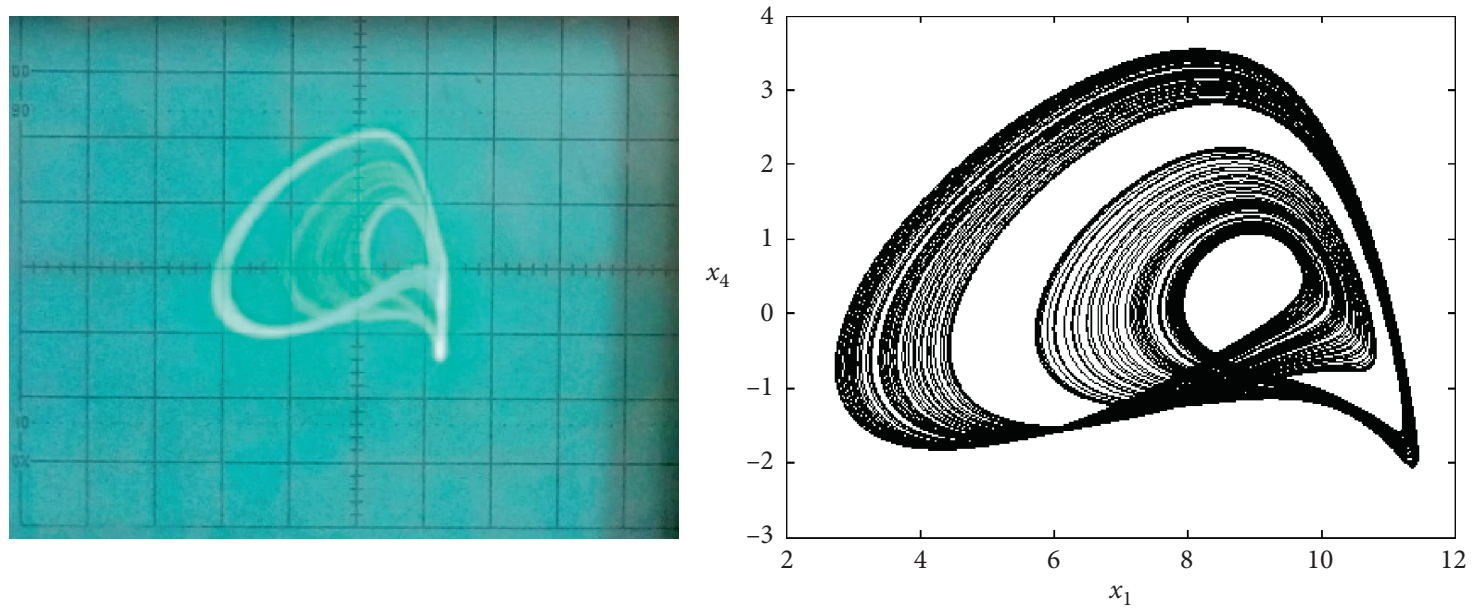

(c)
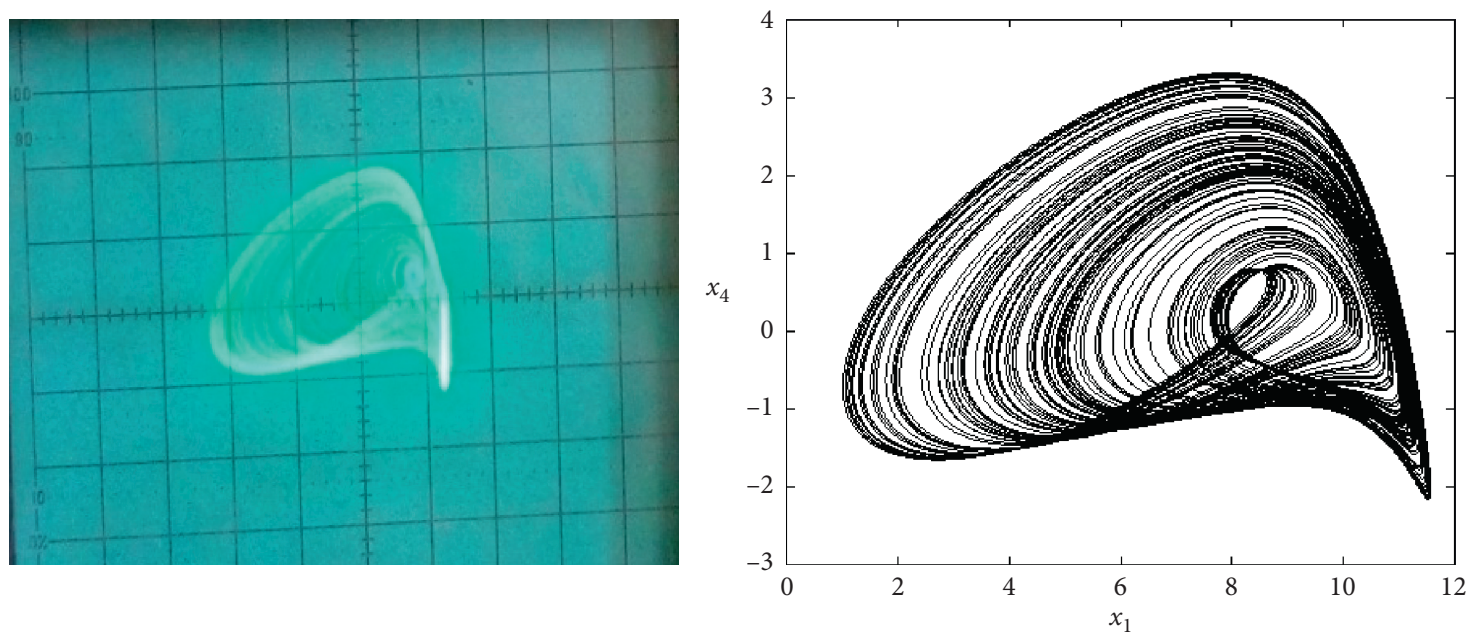

(d)
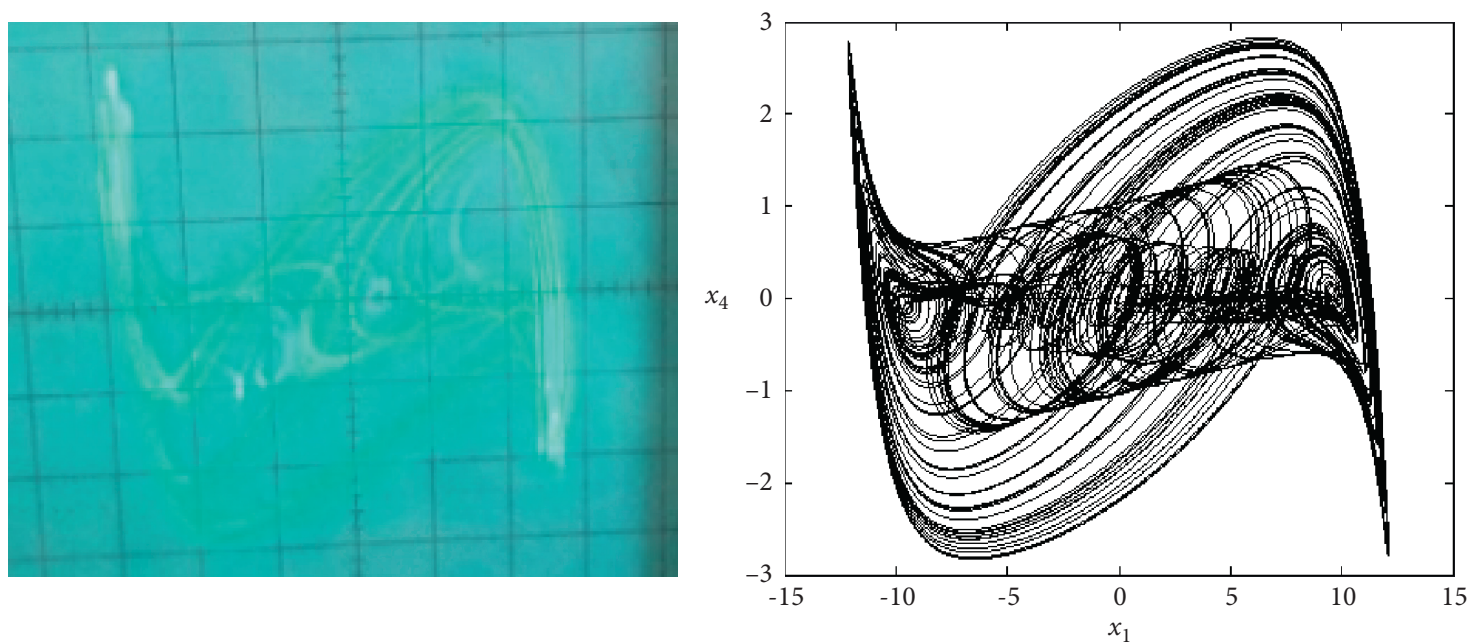

(e)

FIGURE 21: Experimental phase portraits (right column) and corresponding numerical ones (left column) obtained by a direct integration of the system (1) confirming the scenario to chaos in the system for varying Ra (i.e., parameter $a$ ): (a) period- 1 for $R_{a}=1.428$, (b) period-2 for $R_{a}=807$, (c) single-band chaos for $R_{a}=684$, (d) single-band chaos for $R_{a}=500$, and (e) double-band chaos for $R_{a}=333,33$. The scales are $X=0.2 \mathrm{~V} /$ div and $Y=0.5 \mathrm{~V} /$ div. 

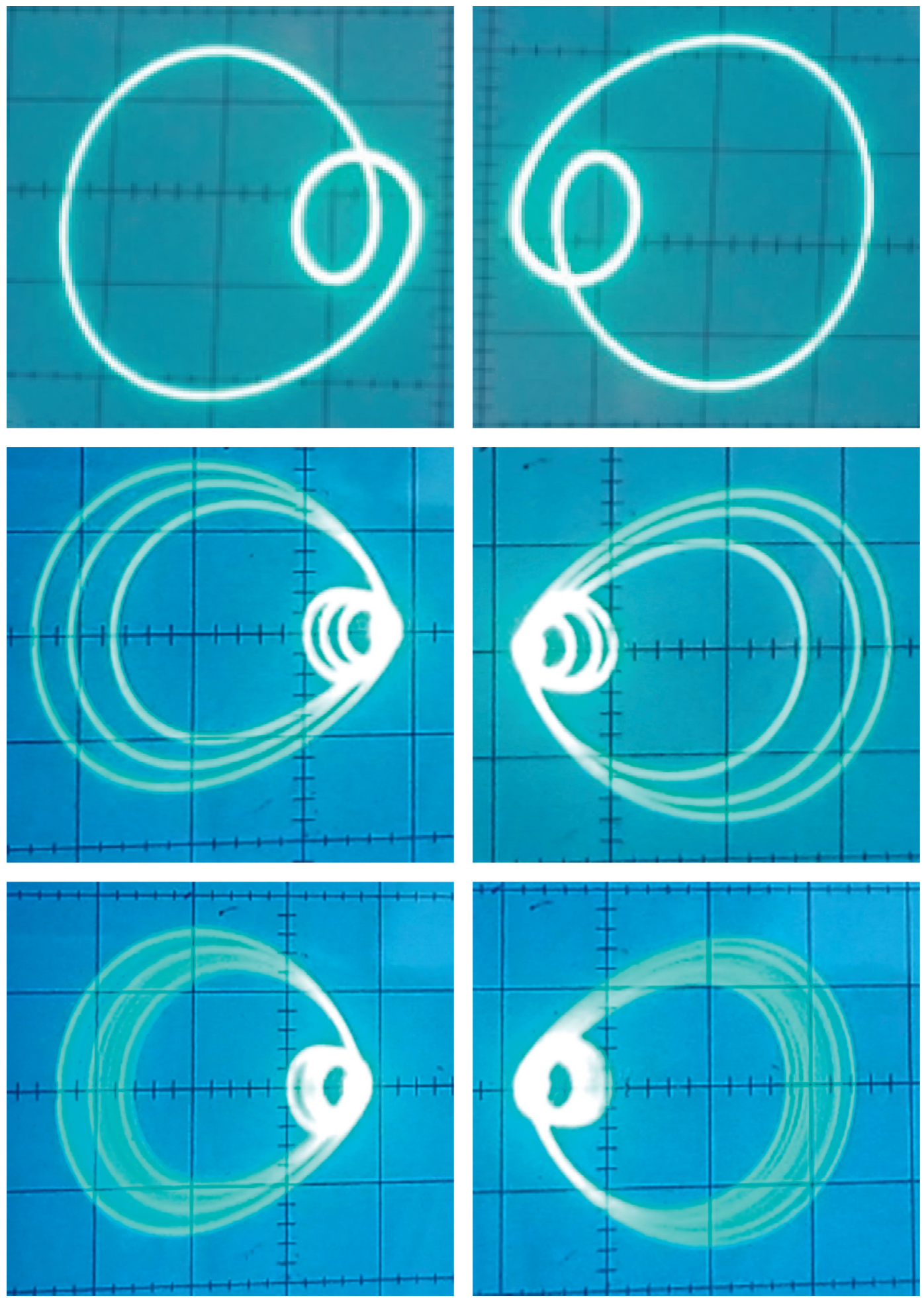

FIGURE 22: Coexistence of multiples attractors for $R_{c}=5.16 \mathrm{k} \Omega$. Both periodic and chaotic attractors appear randomly in the experiment when switching on and off the power supply. The scales are $X=5 \mathrm{~V} / \mathrm{div}$ and $Y=2 \mathrm{~V} / \mathrm{div}$ for all pictures.

coexistence of fixed points, period-2 attractor, and chaotic attractor. Those attractors appear randomly by switching on and off the power supply. We can conclude that the mathematical model proposed in this work perfectly describes the real behavior of the novel hyperjerk circuit.

\section{Conclusion}

This work has proposed and investigated a new chaotic hyperjerk circuit with three equilibrium points having hyperbolic sine nonlinearity. The chaotic behavior observed in the system is due to the nonlinear component formed by two 
antiparallel diodes. Classical nonlinear analysis tools have been used to study the complete dynamics of the system. The bifurcation analysis of the new circuit shows that the chaotic double-band attractor arises from the period-doubling scenario followed by the symmetry recovering crisis event. In addition, some properties of the system such as antimonotonicity and offset boosting have been revealed. In particular, various regions in the parameter space in which the system develops the coexistence of up to ten disconnected attractors consisting of stable fixed points, limit cycles, and strange attractors have been reported. The coexistence of periodic, chaotic, and stable fixed points discovered in this work has not yet been reported in a hyperjerk system (at least as simple as the case discussed) and thus merits dissemination. To validate the theoretical study presented in this work, the new chaotic hyperjerk circuit has been realized and used for the investigations. Experimental results agree well with those obtained during the numerical experiment, thus confirming the feasibility of the proposed model. Owing to its extreme simplicity coupled with extremely rich dynamics, the new hyperjerk circuit introduced in this work has potential utility for information encryption as well as for other chaos-based applications [62].

\section{Data Availability}

No data were used in this study.

\section{Conflicts of Interest}

The authors declare that they have no conflicts of interest.

\section{References}

[1] E. N. Lorenz, "Deterministic nonperiodic flow," Journal of the Atmospheric Sciences, vol. 20, no. 2, pp. 130-141, 1963.

[2] O. E. Rössler, "An equation for continuous chaos," Physics Letters A, vol. 57, no. 5, pp. 397-398, 1976.

[3] G. R. Chen, Controlling Chaos and Bifurcations in Engineering Systems, CRC Press, Boca Raton, FL, USA, 1999.

[4] S. Jafari and J. C. Sprott, "Simple chaotic flows with a line equilibrium," Chaos, Solitons \& Fractals, vol. 57, pp. 79-84, 2013.

[5] V.-T. Pham, C. Volos, S. Jafari, Z. Wei, and X. Wang, "Constructing a novel no-equilibrium chaotic system," International Journal of Bifurcation and Chaos, vol. 24, no. 5, p. 1450073, 2014.

[6] J. Lü and G. Chen, "A new chaotic attractor coined," International Journal of Bifurcation and Chaos, vol. 12, no. 03, pp. 659-661, 2002.

[7] F. Y. Dalkiran and J. C. Sprott, "Simple chaotic hyperjerk system," International Journal of Bifurcation and Chaos, vol. 26, no. 11, p. 1650189, 2016.

[8] S. J. Linz, "Nonlinear dynamical models and jerky motion," American Journal of Physics, vol. 65, no. 6, pp. 523-526, 1997.

[9] J. Kengne, Z. T. Njitacke, A. N. Negou, M. Fouodji Tsostop, and H. B. Fotsin, "Coexistence of multiple attractors and crisis route to chaos in a novel chaotic jerk circuit," International Journal of Bifurcation and Chaos, vol. 26, no. 5, p. 1650081, 2016.

[10] J. Kengne, A. N. Negou, and D. Tchiotsop, "Antimonotonicity, chaos and multiple attractors in a novel autonomous memristor-based jerk circuit," Nonlinear Dynamics, vol. 88, no. 4, pp. 2589-2608, 2017.
[11] J Kengne, V. R. F. Signing, J. C. Che djou, and G. D. Leutcho, Nonlinear behavior of a novel chaotic jerk circuit: antimonotonicity, crisis and multiple coexisting attractors," International Journal of Dynamics and Control, vol. 6, pp. 1-18, 2017.

[12] Z. T. Njitacke, J. Kengne, and L. K. Kengne, "Antimonotonicity, chaos and multiple coexisting attractors in a simple hybrid diode-based jerk circuit," Chaos, Solitons \& Fractals, vol. 105, pp. 77-91, 2017.

[13] P. Daltzis, S. Vaidyanathan, V. T. Pham, C. Volos, E. Nistazakis, and G. Tombras, "Hyperchaotic attractor in a novel hyperjerk system with two nonlinearities," Circuits, Systems, and Signal Processing, vol. 37, no. 2, pp. 613-635, 2018.

[14] J. C. Sprott, Elegant Chaos: Algebraically Simple Chaotic Flows, World Scientific Publishing, Singapore, 2010.

[15] A. P. Kuznetsov, S. P. Kuznetsov, and N. V. Stankevich, "A simple autonomous quasiperiodic self-oscillator," Communications in Nonlinear Science and Numerical Simulation, vol. 15, no. 6, pp. 1676-1681, 2010.

[16] N. V. Kuznetsov, G. A. Leonov, and V. I. Vagaitsev, “Analytical-numerical method for attractor localization of generalized Chua's system*," IFAC Proceedings Volumes, vol. 43, no. 11, pp. 29-33, 2010.

[17] N. V. Kuznetsov, O. A. Kuznetsova, G. A. Leonov, and V. I. Vagaitsev, "Hidden attractor in chua's circuits," in Proceedings of the 8th International Conference Informatics in Control, Automation and Robotics, pp. 279-283, ICINCO, Noordwijkerhout, The Netherland, 2011.

[18] N. V. Kuznetsov, G. A. Leonov, and S. M. Seledzhi, "Hidden oscillations in nonlinear control systems," IFAC Proceedings Volumes, vol. 44, no. 1, pp. 2506-2510, 2011.

[19] G. A. Leonov, N. V. Kuznetsov, and V. I. Vagaitsev, "Hidden attractor in smooth Chua systems," Physica D: Nonlinear Phenomena, vol. 241, no. 18, pp. 1482-1486, 2012.

[20] G. A. Leonov and N. V. Kuznetsov, "Hidden attractors in dynamical systems. From hidden oscillations in hilbertKolmogorov, aizerman, and kalman problems to hidden chaotic attractor in chua circuits," International Journal of Bifurcation and Chaos, vol. 23, no. 1, pp. 1330002-1330011, 2013.

[21] G. D. Leutcho, J. Kengne, and L. K. Kengne, "Dynamical analysis of a novel autonomous 4-D hyperjerk circuit with hyperbolic sine nonlinearity: chaos, antimonotonicity and a plethora of coexisting attractors," Chaos, Solitons \& Fractals, vol. 107, pp. 67-87, 2018.

[22] K. E. Chlouverakis and J. C. Sprott, "Chaotic hyperjerk systems," Chaos, Solitons \& Fractals, vol. 28, no. 3, pp. 739-746, 2006.

[23] V. Sundarapandian, C. Volos, V. T. Pham, and K. Madhavan, "Analysis, adaptive control and synchronization of a novel 4D hyperchaotic hyperjerk system and its SPICE implementation," Archives of Control Sciences, vol. 25, no. 1, pp. 135-158, 2015.

[24] C. Li, "Coexisting hidden attractors in a 4-D simplified Lorenz System," International Journal of Bifurcation and Chaos in Applied Sciences and Engineering, vol. 24, p. 1450034, 2013.

[25] B. Bao, X. Zou, Z. Liu, and F. Hu, "Generalized memory element and chaotic memory system," International Journal of Bifurcation and Chaos, vol. 23, no. 8, p. 1350135, 2013.

[26] S. Ren, S. Panahi, K. Rajagopal, K. Akgul, V. T. Pham, and S. Jafari, "A new chaotic flow with hidden attractor: the first hyperjerk system with no-equilibrium," Zeitschrift für Naturforschung A, vol. 73, 2018. 
[27] S. Vaidyanathan, S. Jafari, V. T. Pham, A. T. Azar, and F. Alsaadi, "A 4-D chaotic hyperjerk system with a hidden attractor, adaptive backstepping control and circuit design"” Archive of Control Sciences, vol. 28, no. 2, pp. 239-254, 2018.

[28] J. Kengne, S. Jafari, and Z. T. Njitacke, "Dynamic analysis and electronic circuit implementation of a novel 3D autonomous system without linear terms," Communications in Nonlinear Science and Numerical Simulation, vol. S1007-5704, no. 17, pp. 30126-30130, 2017.

[29] J. C. Sprott, "A proposed standard for the publication of new chaotic systems," International Journal of Bifurcation and Chaos, vol. 21, no. 9, pp. 2391-2394, 2011.

[30] A. Buscarino, L. Fortuna, M. Frasca, and L. V. Gambuzza, "A chaotic circuit based on Hewlett-Packard memristor," Chaos, vol. 22, no. 2, Article ID 023136, 2012.

[31] M. P. Hanias, G. Giannaris, A. Spyridakis, and A. Rigas, "Time series analysis in chaotic diode resonator circuit," Chaos, Solitons \& Fractals, vol. 27, no. 2, pp. 569-573, 2006.

[32] D. W. Sukow, M. E. Bleich, J. Gauthier, and J. E. S. Socolar, "Controlling chaos in a fast diode resonator using extended time-delay auto-synchronization: experimental observations and theoretical analysis," Chaos, vol. 7, no. 4, pp. 560-576, 1997.

[33] S. H. Strogatz, Nonlinear Dynamics and Chaos, AddisonWesley, Boston, MA, USA, 1994.

[34] N. ah and B. Balachandran, Applied Nonlinear Dynamics: Analytical, Computational and Experimental Methods, John Wiley \& Sons, Hoboken, NJ, USA, 1995.

[35] A. Wolf, J. B. Swift, H. L. Swinney, and J. A. Vastano, "Determining Lyapunov exponents from a time series," Physica D: Nonlinear Phenomena, vol. 16, no. 3, pp. 285-317, 1985.

[36] L. Chunbiao and J. C. Sprott, "An infinite 3-D periodic lattice of chaotic attractors," Physics Letters A, vol. 382, no. 8, pp. 581-587, 2017.

[37] L. Chunbiao, J. C. Sprott, T. Kapitaniak, and T. Lu, "Infinite lattice of hyperchaotic strange attractors," Chaos, Solitons and Fractals, vol. 109, pp. 76-82, 2018.

[38] F. T. Fozin, J. Kengne, and F. B. Pelab, "Dynamical analysis and multistability in autonomous hyperchaotic oscillator with experimental verification," Nonlinear Dynamics, vol. 93, no. 2, pp. 653-669, 2018.

[39] N. Z. Tabekoueng, D. I. Sami, J. Kengne, and Cheukem, "Coexistence of firing patterns and its control in two neurons coupled trough an asymmetric electrical synapse," Chaos, vol. 30, Article ID 023101, 2020.

[40] K. L. Kamdjeu, J. Kengne, P. R. J. Mboupda, and T. T. H. Kamdem, "Dynamics, control and symmetry breaking aspects of an infinite equilibrium chaotic system," International Journal of Dynamics and Control, 2020.

[41] H. Chang, Y. Li, and F. Yuan, "Extreme multistability with hidden attractors in a simple memristor-based circuit," International Journal of Bifurcation and Chaos in Applied Sciences and Engineering, vol. 29, no. 6, 2019.

[42] Q. Lai, C. Chen, X. W. Zhao, J. Kengne, and C. Volos, "Constructing chaotic system with multiple coexisting attractors," IEEE Acesss, vol. 7, 2019.

[43] Q. Lai, K. D. P. Kuate, F. Liu, and H. C. H. Iu, "An extremely simple chaotic system with infinitely many coexisting attractors," IEEE Transactions on Circuits and Systems II: Express Briefs, vol. 1, 2019.

[44] K. Rajagopal, P. J. Singh, K. B. Roy, and A. Karthikeyan, "Dissipative and conservative chaotic nature of a new quasiperiodically forced oscillator with megastability," Chinese Journal of Physics, vol. 58, 2019.
[45] Y. Li and J. Zeng, "A unique jerk system with abundant dynamics: symmetric or asymmetric bistability, tristability, and coexisting bubbles," Brazilian Journal of Physics, vol. 50, 2020.

[46] M. Tuna, A. Karthikeyan, K. Rajagopal, M. Alçın, and I. Koyuncu, "Hyperjerk multiscroll oscillators with megastability: analysis, FPGA implementation and A novel ANNring-based true random number generator," International Journal of Electronics and Communications, vol. 112, 2019.

[47] C. Li, X. Wang, and G. Chen, "Diagnosing multistability by offset boosting," Nonlinear Dynamics, vol. 90, 2017.

[48] C. Li, J. C. Sprott, A. Akgul, H. H. C. Lu, and Y. Zhao, "A new chaotic oscillator with free control," Chaos, vol. 27, Article ID 083101, 2017.

[49] C. Li, J. C. Sprott, and Y. Mei, "An infinite 2-D lattice of strange attractors," Nonlinear Dynamics, vol. 89, 2017.

[50] C. Li, J. C. Sprott, W. Hu, and Y. Xu, "Infinite multistability in a self-reproducing chaotic system," International Journal of Bifurcation and Chaos, vol. 27, no. 10, Article ID 1750160, 2017.

[51] C. Li, J. C. Sprott, T. Kapitaniak, and T. Lu, "Infinite lattice of hyperchaotic strange attractors," Chaos, Solitons \& Fractals, vol. 109 , pp. 76-82, 2018.

[52] C. Li and J. C. Sprott, "An infinite 3-D quasiperiodic lattice of chaotic attractors," Physics Letters A, vol. 382, no. 8, pp. 581-587, 2018.

[53] S. P. Dawson, C. Grebogi, J. A. Yorke, I. Kan, and H. Koçak, "Antimonotonicity: inevitable reversals of period-doubling cascades," Physics Letters A, vol. 162, no. 3, pp. 249-254, 1992.

[54] L. Kocarev, K. S. Halle, K. Eckert, and L. O. Chua, "Experimental observation of antimonotonicity in Chua's circuit," International Journal of Bifurcation and Chaos, vol. 3, no. 4, pp. 1051-1055, 19933.

[55] I. Manimehan and P. Philominathan, "Composite dynamical behaviors in a simple series-parallel LC circuit," Chaos, Solitons \& Fractals, vol. 45, no. 12, pp. 1501-1509, 2012.

[56] I. M. Kyprianidis, I. N. Stouboulos, P. Haralabidis, and T. Bountis, "Antimonotonicity and chaotic dynamics in a fourth-order autonomous nonlinear electric circuit," International Journal of Bifurcation and Chaos, vol. 10, no. 08, pp. 1903-1915, 2000.

[57] S. P. Dawson, C. Grebogi, and H. Koçak, "Mechanism for antimonotonicity in scalar maps with two critical points," Physical Review E, vol. 48, no. 3, pp. 1676-1682, 1993.

[58] V.-T. Pham, A. Akgul, C. Volos, S. Jafari, and T. Kapitaniak, "Dynamics and circuit realization of a no-equilibrium chaotic system with a boostable variable," AEU-International Journal of Electronics and Communications, vol. 78, pp. 134-140, 2017.

[59] E. Tlelo-Cuautle, A. D. Pano-Azucena, J. J. Rangel-Magdaleno, V. H. Carbajal-Gomez, and G. Rodriguez-Gomez, "Generating a 50 -scroll chaotic attractor at $66 \mathrm{MHz}$ by using FPGAs," Nonlinear Dynamics, vol. 85, no. 4, pp. 2143-2157, 2016.

[60] B. Muthuswamy and S Banerjee, A Route to Chaos Using FPGAs, Complexity and Computation Springer International Publishing, Berlin, Germany, 2015.

[61] E. Tlelo-Cuautle, J. J. Rangel-Magdaleno, A. D. Pano-Azucena, P. J. Obeso-Rodelo, and J. C. Nunez-Perez, "FPGA realization of multi-scroll chaotic oscillators," Communications in Nonlinear Science and Numerical Simulation, vol. 27, no. 1-3, pp. 66-80, 2015.

[62] I. Koyuncu, A. T. Ozcerit, and I. Pehlivan, "Implementation of FPGA-based real time novel chaotic oscillator," Nonlinear Dynamics, vol. 77, no. 1-2, pp. 49-59, 2014. 